\title{
Bifurcation from relative periodic solutions
}

\author{
CLAUDIA WULFF $\dagger$, JEROEN S. W. LAMB $\ddagger$ and IAN MELBOURNE $\S$ \\ $\dagger$ Institut für Mathematik I, Freie Universität Berlin, Arnimallee 2-6, 14195 Berlin, \\ Germany \\ $\$$ Department of Mathematics, Imperial College, 180 Queens Gate, London SW7 2BZ, UK \\ (e-mail: jsw.lamb@ic.ac.uk) \\ $\S$ Department of Mathematics, University of Houston, Houston, TX 77204-3476, USA
}

(Received 20 April 1999 and accepted in revised form 1 December 1999)

\begin{abstract}
Relative periodic solutions are ubiquitous in dynamical systems with continuous symmetry. Recently, Sandstede, Scheel and Wulff derived a center bundle theorem, reducing local bifurcation from relative periodic solutions to a finite-dimensional problem. Independently, Lamb and Melbourne showed how to systematically study local bifurcation from isolated periodic solutions with discrete spatiotemporal symmetries.

In this paper, we show how the center bundle theorem, when combined with certain group theoretic results, reduces bifurcation from relative periodic solutions to bifurcation from isolated periodic solutions. In this way, we obtain a systematic approach to the study of local bifurcation from relative periodic solutions.
\end{abstract}

\section{Introduction}

Relative equilibria and relative periodic solutions occur in numerous physical experiments in which there are continuous symmetries present. For example, in excitable media there arise spirals that rigidly rotate, see [36], as well as spirals that undergo quasiperiodic meandering, see [32], and those that undergo linear drift $[\mathbf{2 5}, \mathbf{3 8}]$. The rigidly rotating spirals are examples of relative equilibria-in a rotating frame they are ordinary equilibria. The quasiperiodically meandering and linearly drifting spirals are examples of relative periodic solutions. In appropriate moving frames (a rotating frame and a translating frame, respectively) they reduce to periodic solutions.

Similarly, in the Taylor-Couette experiment, wavy vortices [1] are examples of relative equilibria, whereas modulated wavy vortices [17] are examples of relative periodic solutions. Relative equilibria and relative periodic solutions also arise in flame experiments [16] (and in the associated numerics [3]), and in two-dimensional convection patterns [29].

Transitions from relative equilibria and relative periodic solutions have been analyzed in the above settings using equivariant bifurcation theory. This is an extension to systems 
with symmetry of the standard local bifurcation theory for systems without symmetry. We note that in dynamical systems without symmetry, there is a complete theory of the generic local bifurcations that occur as a single bifurcation parameter is varied; see, for example, Guckenheimer and Holmes [19, Ch. 3]. Local bifurcations are by definition the bifurcations that occur in the neighborhood of a non-hyperbolic equilibrium or a nonhyperbolic periodic solution.

As described in more detail below, there exists a systematic approach to bifurcation from equilibria $[\mathbf{1 5}]$ and relative equilibria $[\mathbf{9 , 2 0 , 3 0}]$ in systems with symmetry. The analogous theory for periodic solutions is due to $[\mathbf{2 1}, \mathbf{2 3}]$. In this paper, we develop a systematic approach to bifurcation from relative periodic solutions, building upon previous work of [31].

The first systematic results in equivariant bifurcation theory were obtained for bifurcation from fully symmetric equilibria under the assumption that the group of symmetries $\Gamma$ is a compact Lie group; see Golubitsky et al [15]. Such equilibria are generically isolated.

A relative equilibrium is a $\Gamma$-orbit in phase space that is also invariant under the flow. If we denote the flow by $\Phi_{t}$, then $u_{0}$ lies on a relative equilibrium if and only if $\Phi_{t}\left(u_{0}\right) \in \Gamma u_{0}$ for all $t$. The simplest example of a relative equilibrium is a group orbit of equilibria. A relative equilibrium on which the flow is periodic is called a rotating wave. In general, the flow on a relative equilibrium is either quasiperiodic or unbounded. The flows on a relative equilibrium were classified algebraically by Field $[\mathbf{1 1}]$ in the case that $\Gamma$ is a compact Lie group and by Ashwin and Melbourne [2] for $\Gamma$ a general finite-dimensional Lie group.

In the case that $\Gamma$ is compact, Krupa [20] showed that, modulo drifts along continuous group orbits, the problem of bifurcation from a relative equilibrium reduces generically to the problem of bifurcation from an isolated equilibrium as studied in [15]. In this way, the usual center manifold theorem for equilibria [19] translates into a center bundle theorem for relative equilibria. Sandstede et al [30] considered linear isometric representations of a possibly non-compact Lie group $\Gamma$ on a Banach space. Suppose that $\Gamma$ acts continuously on $u_{0}$ and that $\Gamma u_{0}$ is an embedded submanifold with compact isotropy subgroup

$$
\Delta=\left\{\gamma \in \Gamma: \gamma u_{0}=u_{0}\right\} .
$$

Under certain spectral hypotheses, it is shown in [30] that a finite-dimensional center bundle reduction still exists, and moreover that $\Gamma$ acts properly on the center bundle. (Recall that $\Gamma$ acts properly on the space $M$ if the map $(\gamma, u) \mapsto(\gamma u, u) \in M \times M$ sends closed sets to closed sets and preimages of points in $M \times M$ are compact.) It is then possible to apply the differentiable slice theorem of Palais [28] (see also the book by Tom Dieck [7]). As shown in Fiedler et al [9], the slice theorem gives convenient coordinates on the center bundle, enabling the computation of the drifts arising through bifurcation.

More precisely, let $\Gamma u_{0}$ be a relative equilibrium with compact isotropy subgroup $\Delta$ and let $V$ be a $\Delta$-invariant cross-section. Consider the free action of $\Gamma \times \Delta$ on $\Gamma \times V$ where $\Gamma$ acts by left multiplication on the $\Gamma$-component, and $\delta \in \Delta$ acts as

$$
\delta \cdot(\gamma, v)=\left(\gamma \delta^{-1}, \delta v\right) .
$$

Since $\Delta$ is compact and acts freely, we can form the quotient manifold

$$
\Gamma \times{ }_{\Delta} V=(\Gamma \times V) / \Delta .
$$


It follows from Palais [28] that there is a neighborhood $U$ of the relative equilibrium that is diffeomorphic to $\Gamma \times_{\Delta} V$. The vector field restricted to $U$ lifts to a $\Gamma \times \Delta$-equivariant vector field on $\Gamma \times V$. See Fiedler et al [9].

Now we turn to bifurcation from relative periodic solutions. Recall that $u_{0}$ lies on a relative periodic solution if $u_{0}$ does not lie on a relative equilibrium and there is some time $T>0$ such that $\Phi_{T}\left(u_{0}\right) \in \Gamma u_{0}$. The corresponding relative periodic solution $\mathcal{P}$ is defined to be

$$
\mathcal{P}=\left\{\gamma \Phi_{t}\left(u_{0}\right): \gamma \in \Gamma, t \in[0, T)\right\}
$$

The minimal choice of $T$ is called the relative period of $\mathcal{P}$ and by rescaling time we may suppose that $T=1$. The flows on a relative periodic solution were classified by Krupa [20] in the case that $\Gamma$ is compact (see also Field [12]) and by Ashwin and Melbourne [2] in the general case.

As in the case of relative equilibria, we suppose that the isotropy subgroup $\Delta$ of the point $u_{0} \in \mathcal{P}$ is compact. In Lamb and Melbourne [21], it is assumed in addition that $\operatorname{dim} \Gamma=\operatorname{dim} \Delta$. The relative periodic solution is then an ordinary periodic solution and moreover is generically isolated. A systematic approach to bifurcation from such isolated periodic solutions is presented in [21]. Once again, the problem is reduced to the problem of bifurcation from an isolated equilibrium.

A center bundle theorem for relative periodic solutions is proved in Sandstede et al [31]. The local dynamics then reduces from an infinite-dimensional phase space to a finitedimensional manifold $M$ on which the Lie group $\Gamma$ acts smoothly and properly. We take this as our starting point and refer to [31] for a statement and discussion of the technical hypotheses behind the center bundle theorem.

Remark 1.1. The center bundle theorem of [31] holds quite generally when $\Gamma$ is compact, and under certain hypotheses when $\Gamma$ is non-compact. Moreover, as shown in [31], in some instances it is possible to choose coordinates on the center bundle so that the reduced finitedimensional equations are amenable to established techniques from equivariant bifurcation theory. However, we emphasize that the issue of choosing such coordinates is not solved in general in [31]. Hence, the results in [31] (even when combined with those in $[\mathbf{2 1}, \mathbf{2 3}]$, and even when $\Gamma$ is compact) fall short of providing a fully systematic theory for bifurcation from relative periodic solutions. Such a theory is the purpose of this paper.

1.1. Spatial and spatiotemporal symmetry. Let $\Gamma$ be a finite-dimensional Lie group acting smoothly and properly on the finite-dimensional manifold $M$. Suppose that $\mathcal{P}$ is a relative periodic solution (with relative period 1 ) for the $\Gamma$-equivariant ODE

$$
\dot{u}=\mathcal{F}(u),
$$

where $\mathcal{F}: M \rightarrow T M$ is a $\Gamma$-equivariant vector field.

The symmetries that leave $\mathcal{P}$ invariant come in two forms. First, there is the group of spatial symmetries, namely the isotropy subgroup $\Delta$ of $u_{0}$. (Since $\Gamma$ acts properly on $M$, the isotropy subgroup $\Delta$ is automatically compact.) Second, there is the group of spatiotemporal symmetries $\Sigma$ defined in the following way. Choose $\sigma \in \Gamma$ such that 
$\Phi_{1}\left(u_{0}\right)=\sigma u_{0}$. Then, $\Sigma$ is the closed subgroup of $\Gamma$ generated by $\Delta$ together with $\sigma$. Note that $\Delta$ is a normal subgroup of $\Sigma$.

The subgroups $\Delta$ and $\Sigma$ depend on $u_{0}$ but are unique up to conjugacy within $\Gamma$. We shall regard $u_{0}$, and hence $\Delta$ and $\Sigma$, as being fixed. More significantly, $\sigma$ is defined only up to multiplication by elements of $\Delta$ (so only the coset $\sigma \Delta$ is uniquely determined). It turns out that particularly convenient choices of $\sigma$ exist when $\Gamma$ is an algebraic group. (More generally, it is sufficient that the normalizer $N(\Delta)$ of $\Delta$ is an algebraic group.) For background material on algebraic groups, we refer to [27]. Such groups are characterized as being those subgroups of $\mathbf{G L}(n, \mathbb{R}), n \geq 1$, that are defined by polynomial equalities in the coefficients of the matrices. Thus, every algebraic group is a finite-dimensional Lie group. We note that all compact Lie groups are algebraic, as are the Euclidean groups $\mathbf{E}(N)$. The classical Lie groups are algebraic, and more generally a semisimple Lie group is an algebraic group if and only if it is a matrix group.

The following result about algebraic groups is proved in $\$ 5$. Let LG denote the Lie algebra of a Lie group $G$.

Proposition 1.2. Suppose that $\Gamma$ is an algebraic group, $\Delta$ is a compact subgroup, and $\sigma_{0} \in N(\Delta)$. Let $\Sigma$ be the closed subgroup of $\Gamma$ generated by $\Delta$ and $\sigma_{0}$. Then there is an element $\sigma \in \sigma_{0} \Delta$ such that $\sigma^{n} \in \exp \operatorname{LZ}(\Sigma)$ for some $n \geq 1$.

Here, $Z(\Sigma)$ is the centralizer of $\Sigma$ inside $\Gamma$.

1.2. Skew product for relative periodic solutions. Suppose that $\mathcal{P}$ is a relative periodic solution for a $\Gamma$-equivariant vector field on $M$. As before, the spatiotemporal symmetry $\Sigma$ is the closed subgroup of $\Gamma$ generated by the spatial symmetry $\Delta$ together with an element $\sigma$.

Suppose further that $\Gamma$ is algebraic and $\Delta$ is compact. By Proposition 1.2, we may assume without loss that $\sigma^{n}=\exp n \xi$ where $n \geq 1, \xi \in \mathrm{L} Z(\Sigma)$. We form a semidirect product $\Delta \rtimes \mathbb{Z}_{2 n}$ by adjoining to $\Delta$ an element $Q$ of order $2 n$, where $Q \delta Q^{-1}=\sigma \delta \sigma^{-1}$ for $\delta \in \Delta$. (In particular, $Q^{n}$ lies in the center of $\Delta \rtimes \mathbb{Z}_{2 n}$.) Also, define $\alpha=\exp (-\xi) \sigma$, so $\alpha^{n}=$ id

We now state our main result. The proof is given in $\S 2$.

THEOREM 1.3. In a comoving frame, moving uniformly with velocity $\xi$ :

(a) there is a neighborhood $U$ of the relative periodic solution $\mathcal{P}$ such that

$$
U \cong \frac{\left(\Gamma \times{ }_{\Delta} V\right) \times S^{1}}{\mathbb{Z}_{2 n}} \cong \frac{\Gamma \times V \times S^{1}}{\Delta \rtimes \mathbb{Z}_{2 n}},
$$

where $V$ is a representation of the group $\Delta \rtimes \mathbb{Z}_{2 n}, S^{1}=\mathbb{R} / 2 n \mathbb{Z}$, and the action of $\Delta \rtimes \mathbb{Z}_{2 n}$ on $\Gamma \times V \times S^{1}$ is given by

$$
\begin{gathered}
\delta \cdot(\gamma, v, \theta)=\left(\gamma \delta^{-1}, \delta v, \theta\right), \quad \delta \in \Delta, \\
Q \cdot(\gamma, v, \theta)=\left(\gamma \alpha^{-1}, Q v, \theta+1\right) ;
\end{gathered}
$$

(b) the equations on U lift to $\Delta \rtimes \mathbb{Z}_{2 n}$-equivariant skew product equations on $\Gamma \times V \times S^{1}$ of the form (after reparameterizing time)

$$
\dot{\gamma}=\gamma f_{\Gamma}(v, \theta), \quad \dot{v}=f_{V}(v, \theta), \quad \dot{\theta}=1,
$$


where $f_{\Gamma}: V \times S^{1} \rightarrow \mathrm{L} \Gamma$ and $f_{V}: V \times S^{1} \rightarrow V$ are smooth vector fields satisfying $f_{\Gamma}(0, \theta)=\xi, f_{V}(0, \theta)=0$, for all $\theta \in S^{1}$.

Remark 1.4. (a) In the comoving frame, the relative periodic solution $\mathcal{P}$ is transformed into a group orbit (under $\Gamma$ ) of ordinary periodic solutions. We speak of comoving frames since it is necessary to pass both to corotating frames (in case $\mathcal{P}$ is a modulated rotating wave) and to cotraveling frames (in case $\mathcal{P}$ is a modulated traveling wave).

(b) The vector space $V$ in Theorem 1.3 is defined to be a $\Delta$-invariant cross-section to the relative periodic solution $\mathcal{P}$, see [31] and also $\S 2$. The representation of $\Delta \rtimes \mathbb{Z}_{2 n}$ is arbitrary, in the sense that any representation can arise for an appropriate choice of manifold $M$ and vector field $\mathcal{F}$. Furthermore, $f_{\Gamma}: V \times S^{1} \rightarrow \mathrm{L} \Gamma$ and $f_{V}: V \times S^{1} \rightarrow V$ are general vector fields satisfying the equivariance conditions and the restrictions at $v=0$ in Theorem 1.3.

(c) If we replace $\Gamma \times V \times S^{1}$ by $\Gamma \times V$, and $\Delta \rtimes \mathbb{Z}_{2 n}$ by $\Delta$, then the skew product structure in Theorem 1.3 reduces to the skew product structure in Fiedler et al [9]. Hence Theorem 1.3 is a generalization to relative periodic solutions of the results in [9] for relative equilibria.

The restrictions on $f_{\Gamma}$ and $f_{V}$ that arise from the action (1.2) of $\Delta \rtimes \mathbb{Z}_{2 n}$ are easily computed to be as follows:

$$
\begin{array}{cll}
f_{\Gamma}(\delta v, \theta)=\operatorname{Ad}_{\delta} f_{\Gamma}(v, \theta), & \delta \in \Delta, & f_{\Gamma}(Q v, \theta)=\operatorname{Ad}_{\alpha} f_{\Gamma}(v, \theta-1), \\
f_{V}(\delta v, \theta)=\delta f_{V}(v, \theta), & \delta \in \Delta, & f_{V}(Q v, \theta)=Q f_{V}(v, \theta-1) .
\end{array}
$$

If time is not reparameterized, the $\dot{\theta}$ equation takes the form $\dot{\theta}=f_{\Theta}(v, \theta)$ where

$$
f_{\Theta}(\delta v, \theta)=f_{\Theta}(v, \theta), \quad \delta \in \Delta, \quad f_{\Theta}(Q v, \theta)=f_{\Theta}(v, \theta-1) .
$$

1.3. Bifurcations. Lamb and Melbourne [21] give a systematic approach to generic bifurcation from isolated periodic solutions with spatiotemporal symmetry. It follows from Theorem 1.3 that this theory generalizes to arbitrary relative periodic solutions provided the hypotheses of Proposition 1.2 are satisfied. Indeed the relative periodic solution $\mathcal{P}$ in Theorem 1.3 reduces to an isolated periodic solution for the $(v, \theta)$-subsystem. Moreover, by Remark 1.4(b), the $(v, \theta)$-subsystem is a general $\Delta \rtimes \mathbb{Z}_{2 n}$-equivariant ODE possessing a periodic solution with spatial symmetry $\Delta$ and spatiotemporal symmetry $\Delta \rtimes \mathbb{Z}_{2 n}$. Hence the theory of [21] applies with the group $\Gamma$ replaced by the group $\Delta \rtimes \mathbb{Z}_{2 n}$. In particular, modulo drifts along continuous group orbits in $\Gamma$ governed by the $\dot{\gamma}$ equation, bifurcation from a relative periodic solution reduces to bifurcation from a periodic solution.

The group $\Delta \rtimes \mathbb{Z}_{2 n}$ is closely related to the group $\Delta \rtimes \mathbb{Z}_{2 k}$ used in [21] to study bifurcation from isolated periodic solutions. There, the integer $k$ is the least positive integer such that $\sigma^{k} \in Z(\Delta)$. Of course, $k$ divides $n$. In fact, it turns out that in applying Theorem 1.3 it is sufficient to consider representations $V$ of $\Delta \rtimes \mathbb{Z}_{2 n}$ in which the generator $Q$ of $\mathbb{Z}_{2 n}$ satisfies $Q^{2 k}=I$.

In other words, the $(v, \theta)$-subsystem in Theorem 1.3 can be taken to be a general $\Delta \rtimes \mathbb{Z}_{2 k}$-equivariant ODE on $V \times S^{1}$ where $S^{1}=\mathbb{R} / 2 k \mathbb{Z}$.

The paper is organized as follows. In $\S 2$, we sketch the results of [31] and prove Theorem 1.3. In $\S 3$, we show how to apply the results in $\S 2$. In $\S 4$, we investigate in 
more depth the structure of relative periodic solutions that arise in equivariant dynamical systems, building upon work of $[\mathbf{2}, \mathbf{1 2}, \mathbf{2 0}]$. In $\S 5$, we prove Proposition 1.2, as well as related results for non-algebraic groups. In $\S 6$, we prove that provided the group action is proper, bifurcation from relative periodic solutions for any symmetry group $\Gamma$ reduces, modulo drifts along continuous group orbits, to bifurcation from isolated periodic solutions as studied in [21] (even though Theorem 1.3 does not hold in this generality).

\section{Relative periodic solutions and skew products}

In this section, we prove the main result, Theorem 1.3, stated in the introduction. In §2.1, we prove a result about twisted equivariant maps. In $\$ 2.2$, we review the skew product construction of Sandstede et al [31]. Theorem 1.3 is proved in $\$ 2.3$. The results in $\S \S 2.1$ and 2.2 hold for general finite-dimensional Lie groups $\Gamma$, whereas in $\$ 2.3$ we suppose that $\Gamma$ is algebraic.

2.1. Twisted equivariant linear maps. Suppose that $\Gamma$ is a finite-dimensional Lie group with compact subgroup $\Delta$. Let $\sigma \in N(\Delta)$. Then $\sigma$ induces an automorphism $\phi \in \operatorname{Aut}(\Delta)$ defined by

$$
\phi(\delta)=\sigma^{-1} \delta \sigma .
$$

Suppose that $\Delta$ acts orthogonally on a finite-dimensional vector space $X$. Following [21], we say that a linear map $L: X \rightarrow X$ is twisted equivariant if

$$
L \delta=\phi(\delta) L
$$

for all $\delta \in \Delta$. (In [24], such a map is said to be $k$-symmetric where $k$ is least such that $\phi^{k}$ is the identity automorphism of $\Delta$.)

PROPOSITION 2.1. Suppose that $L: X \rightarrow X$ is a twisted equivariant non-singular linear map. Then there is a twisted equivariant orthogonal map $A: X \rightarrow X$ such that $A^{-1} L$ is $\Delta$-equivariantly isotopic to the identity.

Proof. By polar decomposition, we can write $L$ uniquely as $L=A B$ where $A$ is orthogonal and $B$ is positive definite. Indeed, $B$ is defined as the unique positive definite square root of $B^{2}=L^{\mathrm{T}} L$.

We claim that $B$ is equivariant and $A$ is twisted equivariant. It follows from the orthogonality of the action of $\Delta$ on $X$ that $L^{\mathrm{T}} L$ commutes with $\Delta$. But then, for each $\delta \in \Delta$, the positive definite matrix $B^{\prime}=\delta B \delta^{-1}$ satisfies $\left(B^{\prime}\right)^{2}=L^{\mathrm{T}} L$. Since $B$ is the unique positive definite square root of $L^{\mathrm{T}} L$, it follows that $B=B^{\prime}=\delta B \delta^{-1}$ so that $B$ commutes with $\Delta$. Since $L$ is twisted equivariant and $B$ is equivariant, we have that $A=L B^{-1}$ is twisted equivariant, proving the claim.

Since $B$ is positive definite and equivariant, it follows that $J_{t}=(1-t) I+t B$ is positive definite and equivariant for all $t \in[0,1]$. In particular, $B$ is equivariantly isotopic to the identity. 
LEMMA 2.2. Let $\Delta$ be a compact Lie group acting orthogonally on $X$ and suppose that $\phi \in \operatorname{Aut}(\Delta)$ is an automorphism of finite order $k$. Let $L: X \rightarrow X$ be a twisted equivariant non-singular linear map. Then there is a twisted equivariant orthogonal map $A: X \rightarrow X$ such that $A^{2 k}=I$ and $A^{-1} L$ is $\Delta$-equivariantly isotopic to the identity.

Proof. By Proposition 2.1, we may first reduce to the case where $L$ is orthogonal.

The map $L^{k}$ is equivariant in the usual sense. Let $\mu$ denote an eigenvalue of $L^{k}$ and denote the corresponding eigenspace by $E_{\mu}$. Note that $E_{\mu}$ is $\Delta$-invariant and is also invariant under $L$. To prove the lemma, it is sufficient to restrict to $E_{\mu}$.

If $\mu= \pm 1$, then we have $L^{2 k}=I$ and it suffices to take $A=L$. Otherwise, $\mu$ lies on the unit circle, but $\mu$ is not real. Let $\lambda \in \mathbb{C}$ denote a $k$ th root of $\mu$ and define $A=\lambda^{-1} L$. Then $A$ is twisted equivariant, and $A^{-1} L$ is equivariantly isotopic to the identity. Moreover, $A^{k}=I$.

2.2. The skew product construction of [31]. We consider a finite-dimensional Lie group $\Gamma$ acting smoothly and properly on a finite-dimensional manifold $M$. Let $\mathcal{F}$ be a $\Gamma$ equivariant vector field on $M$ with flow $\Phi_{t}$ and suppose that $\mathcal{P}$ is a relative periodic solution. Choose $u_{0} \in \mathcal{P}$. Without loss, we have that $\Phi_{1}\left(u_{0}\right)=\sigma u_{0}$ for some $\sigma \in \Gamma$ and $\Phi_{t}\left(u_{0}\right) \notin \Gamma u_{0}$ for $0<t<1$. Let $\Delta$ and $\Sigma$ denote the spatial and spatiotemporal symmetry groups of $\mathcal{P}$, so $\Delta$ is compact and $\Sigma$ is the closed subgroup of $\Gamma$ generated by $\Delta$ and $\sigma$.

In Sandstede et al [31], it was shown how the flow in a neighborhood $U$ of the relative periodic solution $\mathcal{P}$ can be written as a skew product flow on a space of the form $\Gamma \times V \times \mathbb{R}$. Here, $V$ is a $\Delta$-invariant cross-section to $\mathcal{P}$ and $\theta \in \mathbb{R}$ plays the role of the phase along the relative periodic solution. Note that $V$ is a Poincaré section which is transverse to (the Cartesian product of) the time orbit and the group orbit of $u_{0}$.

Roughly speaking, the idea in [31] is to construct a family of cross-sections $V_{\theta}$ around the relative periodic solution and to use the linearized flow $\left(\mathrm{D} \Phi_{\theta}\right)_{u_{0}}$ to define coordinates on $V_{\theta}$. First, write

$$
T_{\Phi_{\theta}\left(u_{0}\right)} M=T_{\Phi_{\theta}\left(u_{0}\right)} \mathcal{P} \oplus V_{\theta},
$$

where $V_{0}=V$ and the cross-sections $V_{\theta}$ are $\Delta$-invariant and depend smoothly on $\theta$. Let $P_{\theta}: T_{\Phi_{\theta}\left(u_{0}\right)} M \rightarrow V_{\theta}$ denote the associated family of projections. By construction, the projections are $\Delta$-equivariant and depend smoothly on $\theta$. Moreover, $\left(\mathrm{D} \Phi_{\theta}\right)_{u_{0}}$ restricts to a $\Delta$-equivariant map $P_{\theta}\left(\mathrm{D} \Phi_{\theta}\right)_{u_{0}}: V \rightarrow V_{\theta}$.

The idea of the proof can be seen by glancing at the submersion $\tau$ defined below in equation (2.3), but with the term $J_{\theta}$ omitted. For technical reasons, we require the following lemma.

LEMMA 2.3. Let $L=P_{0} \sigma^{-1}\left(\mathrm{D} \Phi_{1}\right)_{u_{0}}: V \rightarrow V$.

(a) There is an orthogonal map $A: V \rightarrow V$ and a smooth family of $\Delta$-equivariant non-singular linear maps $J_{\theta}: V \rightarrow V, \theta \in \mathbb{R}$, such that

$$
J_{0}=I, \quad L J_{\theta+1}=J_{\theta} A, \quad \theta \in \mathbb{R} .
$$


(b) The projections $P_{\theta}$ can be chosen so that

$$
P_{\theta+1} \sigma=\sigma P_{\theta}, \quad \theta \in \mathbb{R} .
$$

Moreover,

$$
P_{\theta+1}\left(\mathrm{D} \Phi_{\theta+1}\right)_{u_{0}}=\sigma P_{\theta}\left(\mathrm{D} \Phi_{\theta}\right)_{u_{0}} L
$$

Proof. Note that $L: V \rightarrow V$ is twisted equivariant as defined in $\S 2.1$. That is, $L \delta=\phi(\delta) L$ for all $\delta \in \Delta$, where $\phi \in \operatorname{Aut}(\Delta)$ is given by $\phi(\delta)=\sigma^{-1} \delta \sigma$. It follows from Proposition 2.1 that there is a twisted equivariant orthogonal map $A: V \rightarrow V$ such that $A^{-1} L$ is $\Delta$-equivariantly isotopic to the identity. In particular, there is a smooth family of $\Delta$-equivariant non-singular linear maps $J_{\theta}: V \rightarrow V, \theta \in[0,1]$, such that $J_{0}=I$ and $L J_{1}=A$. This family extends uniquely to a continuous family $J_{\theta}, \theta \in \mathbb{R}$, satisfying equation (2.2). Modifying $J_{\theta}$ near $\theta=0$ and $\theta=1$, we obtain a smooth family $J_{\theta}, \theta \in \mathbb{R}$, proving part (a).

Next, we prove part (b). Let $\langle\cdot, \cdot\rangle$ be a $\Delta$-invariant inner product on $T_{u_{0}} M$ and define $P_{0}$ to be the orthogonal projection onto $V$. We repeat the argument above, but applied to the twisted equivariant map $\widetilde{L}=\sigma^{-1}\left(\mathrm{D} \Phi_{1}\right)_{u_{0}}: T_{u_{0}} M \rightarrow T_{u_{0}} M$ to obtain an orthogonal map $\widetilde{A}: T_{u_{0}} M \rightarrow T_{u_{0}} M$ and a smooth family of $\Delta$-equivariant non-singular linear maps $\widetilde{J}_{\theta}: T_{u_{0}} M \rightarrow T_{u_{0}} M$ such that

$$
\widetilde{J}_{0}=I, \quad \widetilde{L} \widetilde{J}_{\theta+1}=\widetilde{J}_{\theta} \widetilde{A}, \quad \theta \in \mathbb{R} .
$$

Let $\langle\cdot, \cdot\rangle_{\theta}$ be the $\Delta$-invariant inner product on $T_{\Phi_{\theta}\left(u_{0}\right)} M$ defined by

$$
\langle v, w\rangle_{\theta}=\left\langle\widetilde{J}_{\theta}^{-1}\left(\mathrm{D} \Phi_{\theta}\right)_{u_{0}}^{-1} v, \widetilde{J}_{\theta}^{-1}\left(\mathrm{D} \Phi_{\theta}\right)_{u_{0}}^{-1} w\right\rangle .
$$

We compute that

$$
\begin{aligned}
\left(\mathrm{D} \Phi_{\theta+1}\right)_{u_{0}} & =\left(\mathrm{D} \Phi_{\theta}\right)_{\Phi_{1}\left(u_{0}\right)}\left(\mathrm{D} \Phi_{1}\right)_{u_{0}}=\left(\mathrm{D} \Phi_{\theta}\right)_{\sigma u_{0}}\left(\mathrm{D} \Phi_{1}\right)_{u_{0}} \\
& =\sigma\left(\mathrm{D} \Phi_{\theta}\right)_{u_{0}} \sigma^{-1}\left(\mathrm{D} \Phi_{1}\right)_{u_{0}}=\sigma\left(\mathrm{D} \Phi_{\theta}\right)_{u_{0}} \widetilde{L}
\end{aligned}
$$

Hence

$$
\widetilde{J}_{\theta+1}^{-1}\left(\mathrm{D} \Phi_{\theta+1}\right)_{u_{0}}^{-1}=\widetilde{J}_{\theta+1}^{-1} \widetilde{L}^{-1}\left(\mathrm{D} \Phi_{\theta}\right)_{u_{0}}^{-1} \sigma^{-1}=\widetilde{A}^{-1} \widetilde{J}_{\theta}^{-1}\left(\mathrm{D} \Phi_{\theta}\right)_{u_{0}}^{-1} \sigma^{-1}
$$

Using the orthogonality of $\widetilde{A}$, it follows that

$$
\begin{aligned}
\langle v, w\rangle_{\theta+1} & =\left\langle\widetilde{J}_{\theta+1}^{-1}\left(\mathrm{D} \Phi_{\theta+1}\right)_{u_{0}}^{-1} v, \widetilde{J}_{\theta+1}^{-1}\left(\mathrm{D} \Phi_{\theta+1}\right)_{u_{0}}^{-1} w\right\rangle \\
& =\left\langle\widetilde{A}^{-1} \widetilde{J}_{\theta}^{-1}\left(\mathrm{D} \Phi_{\theta}\right)_{u_{0}}^{-1} \sigma^{-1} v, \widetilde{A}^{-1} \widetilde{J}_{\theta}^{-1}\left(\mathrm{D} \Phi_{\theta}\right)_{u_{0}}^{-1} \sigma^{-1} w\right\rangle \\
& =\left\langle\widetilde{J}_{\theta}^{-1}\left(\mathrm{D} \Phi_{\theta}\right)_{u_{0}}^{-1} \sigma^{-1} v, \widetilde{J}_{\theta}^{-1}\left(\mathrm{D} \Phi_{\theta}\right)_{u_{0}}^{-1} \sigma^{-1} w\right\rangle=\left\langle\sigma^{-1} v, \sigma^{-1} w\right\rangle_{\theta}
\end{aligned}
$$

Define $V_{\theta}$ to be the orthogonal complement to $T_{\Phi_{\theta}\left(u_{0}\right)} \mathcal{P}$ in $T_{\Phi_{\theta}\left(u_{0}\right)} M$ with respect to the inner product $\langle\cdot, \cdot\rangle_{\theta}$, and let $P_{\theta}: T_{\Phi_{\theta}\left(u_{0}\right)} M \rightarrow V_{\theta}$ be the orthogonal projection. Then we have $P_{\theta+1} \sigma=\sigma P_{\theta}$ as required.

Finally, we compute that

$$
\begin{aligned}
P_{\theta+1}\left(\mathrm{D} \Phi_{\theta+1}\right)_{u_{0}} & =P_{\theta+1} \sigma\left(\mathrm{D} \Phi_{\theta}\right)_{u_{0}} \sigma^{-1}\left(\mathrm{D} \Phi_{1}\right)_{u_{0}}=\sigma P_{\theta}\left(\mathrm{D} \Phi_{\theta}\right)_{u_{0}} \sigma^{-1}\left(\mathrm{D} \Phi_{1}\right)_{u_{0}} \\
& =\sigma P_{\theta}\left(\mathrm{D} \Phi_{\theta}\right)_{u_{0}} P_{0} \sigma^{-1}\left(\mathrm{D} \Phi_{1}\right)_{u_{0}}=\sigma P_{\theta}\left(\mathrm{D} \Phi_{\theta}\right)_{u_{0}} L,
\end{aligned}
$$

where we have used the fact that $P_{\theta}\left(\mathrm{D} \Phi_{\theta}\right)_{u_{0}}\left(I-P_{0}\right)=0$ (since $\left(\mathrm{D} \Phi_{\theta}\right)\left(T_{u_{0}} \mathcal{P}\right) \subset$ $\left.T_{\Phi_{\theta}\left(u_{0}\right)} \mathcal{P}\right)$. 
Now, consider the submersion $\tau: \Gamma \times V \times \mathbb{R} \rightarrow M$ defined by

$$
\tau(\gamma, v, \theta)=\gamma\left(\Phi_{\theta}\left(u_{0}\right), P_{\theta}\left(\mathrm{D} \Phi_{\theta}\right)_{u_{0}} J_{\theta} v\right) .
$$

It follows from $\Delta$-equivariance of $P_{\theta},\left(\mathrm{D} \Phi_{\theta}\right)_{u_{0}}$ and $J_{\theta}$, and $\Delta$-invariance of the points $\Phi_{\theta}\left(u_{0}\right) \in \mathcal{P}$, that $\tau(\gamma \delta, v, \theta)=\tau(\gamma, \delta v, \theta)$ for all $\delta \in \Delta$. By Lemma 2.3, we have

$$
\begin{aligned}
\tau(\gamma, v, \theta+1) & =\gamma\left(\Phi_{\theta+1}\left(u_{0}\right), P_{\theta+1}\left(\mathrm{D} \Phi_{\theta+1}\right)_{u_{0}} J_{\theta+1} v\right) \\
& =\gamma\left(\sigma \Phi_{\theta}\left(u_{0}\right), \sigma P_{\theta}\left(\mathrm{D} \Phi_{\theta}\right)_{u_{0}} L J_{\theta+1} v\right) \\
& =\gamma \sigma\left(\Phi_{\theta}\left(u_{0}\right), P_{\theta}\left(\mathrm{D} \Phi_{\theta}\right)_{u_{0}} J_{\theta} A v\right)=\tau(\gamma \sigma, A v, \theta) .
\end{aligned}
$$

Define the orthogonal map $Q=A^{-1}$. To summarize, we have the identifications

$$
\tau(\gamma \delta, v, \theta)=\tau(\gamma, \delta v, \theta), \quad \delta \in \Delta, \quad \tau(\gamma, v, \theta+1)=\tau\left(\gamma \sigma, Q^{-1} v, \theta\right)
$$

Remark 2.4. If the term $J_{\theta}$ is absent in the definition (2.3) of $\tau$, then the presence of contracting and expanding eigenvalues for $L$ may imply that the image of $\tau$ is a nonuniform neighborhood of $\mathcal{P}$ as $\theta \rightarrow \pm \infty$.

Next, we introduce an action of a group $\Gamma \times(\Delta \rtimes \mathbb{Z})$ on $\Gamma \times V \times \mathbb{R}$ where $\Gamma$ acts as left multiplication on the $\Gamma$ component and the action of $\Delta \rtimes \mathbb{Z}$ is given by

$$
(\gamma, v, \theta) \mapsto\left(\gamma \delta^{-1}, \delta v, \theta\right), \quad \delta \in \Delta, \quad(\gamma, v, \theta) \mapsto\left(\gamma \sigma^{-1}, Q v, \theta+1\right) .
$$

It is immediate that $\tau$ is $\Gamma$-equivariant. It follows from the identifications (2.4) that $\tau$ induces a $\Gamma$-equivariant map

$$
\tau: \frac{\Gamma \times V \times \mathbb{R}}{\Gamma \times(\Delta \rtimes \mathbb{Z})} \rightarrow M
$$

As shown in [31], this is an equivariant diffeomorphism onto a uniform neighborhood $U$ of the relative periodic solution.

The $\Gamma$-equivariant vector field on the neighborhood $U$ lifts to a $\Gamma \times(\Delta \rtimes \mathbb{Z})$-equivariant vector field on $\Gamma \times V \times \mathbb{R}$. The $\Gamma$-equivariance is equivalent to saying that the lifted vector field has the skew product form

$$
\dot{\gamma}=\gamma f_{\Gamma}(v, \theta), \quad \dot{v}=f_{V}(v, \theta), \quad \dot{\theta}=f_{\Theta}(v, \theta),
$$

where $f_{\Gamma}(0, \theta)=0, f_{V}(0, \theta)=0$, for all $\theta \in \mathbb{R}$.

We end this subsection by demonstrating that the skew product equations (2.5) are general equations satisfying the equivariance conditions and the restrictions at $v=0$ (cf. Remark 1.4(b)). We continue to suppose that $\mathcal{F}: M \rightarrow T M$ is a fixed $\Gamma$-equivariant vector field with flow $\Phi_{t}$ and relative periodic solution $\mathcal{P}$ of relative period 1. As usual, we fix a point $u_{0} \in \mathcal{P}$ and write $\Phi_{1}\left(u_{0}\right)=\sigma u_{0}$. As described in this subsection, we construct a submersion $\tau: \Gamma \times V \times \mathbb{R} \rightarrow U$ where $U$ is a uniform neighborhood of $\mathcal{P}$. Note that $\tau$ depends on the flow $\Phi_{t}$ and hence on the underlying vector field $\mathcal{F}$.

Now, let $\widetilde{\mathcal{F}}: U \rightarrow T U$ be a general $\Gamma$-equivariant vector field defined on the neighborhood $U$ of the relative periodic solution $\mathcal{P}$ and satisfying $\left.\widetilde{\mathcal{F}}\right|_{\mathcal{P}}=\left.\mathcal{F}\right|_{\mathcal{P}}$. The fixed submersion $\tau$ (defined in terms of $\mathcal{F}$ ) gives a one-to-one correspondence between the 
$\Gamma$-equivariant vector fields $\widetilde{\mathcal{F}}: U \rightarrow T U$ with relative periodic solution $\mathcal{P}$ and $\Gamma \times(\Delta \rtimes \mathbb{Z})$ equivariant skew product equations

$$
\dot{\gamma}=\gamma \widetilde{f}_{\Gamma}(v, \theta), \quad \dot{v}=\widetilde{f}_{V}(v, \theta), \quad \dot{\theta}=\widetilde{f}_{\Theta}(v, \theta),
$$

where $\widetilde{f}_{\Gamma}(0, \theta)=0, \widetilde{f}_{V}(0, \theta)=0$, for all $\theta \in \mathbb{R}$.

Hence, by perturbing the underlying vector field $\mathcal{F}$, but keeping the submersion $\tau$ fixed, we obtain arbitrary equivariant perturbations of the skew product equations. It is in this sense that equations (2.5) are general.

2.3. Proof of Theorem 1.3. Now, suppose that $\Gamma$ is algebraic. By Proposition 1.2, we may arrange that $\sigma^{n} \in \exp \operatorname{LZ}(\Sigma)$ for some $n \geq 1$. We make two modifications to the construction in $\$ 2.2$.

The first modification involves the choice of $Q$. Since $\sigma^{n} \in Z(\Delta)$, the induced automorphism $\phi \in \operatorname{Aut}(\Delta)$ satisfies $\phi^{n}=$ Id. It follows from Lemma 2.2 that the isotopy $J_{\theta}$ can be chosen so that the orthogonal map $A=Q^{-1}$ in equation (2.2) has finite order, indeed $Q^{2 n}=I$. In the remainder of this subsection, $\tau: \Gamma \times V \times \mathbb{R} \rightarrow M$ denotes the submersion in $\$ 2.2$ but with the new isotopy $J_{\theta}$ and orthogonal map $Q$.

The second modification is to pass to a convenient comoving frame. Write $\sigma^{n}=\exp n \xi$ where $\xi \in \operatorname{L} Z(\Sigma)$. Define $\alpha=\exp (-\xi) \sigma$, so that $\alpha$ has order $n$. We define the new submersion

$$
\tau^{\text {new }}(\gamma, v, \theta)=\tau(\gamma \exp (-\theta \xi), v, \theta)
$$

Note that $\tau^{\text {new }}$ remains $\Gamma$-equivariant, since $\Gamma$ acts on the left.

Since $\tau(\gamma \delta, v, \theta)=\tau(\gamma, \delta v, \theta)$ for all $\delta \in \Delta$, and $\xi \in \operatorname{LZ}(\Delta)$, it is immediate that $\tau^{\text {new }}(\gamma \delta, v, \theta)=\tau^{\text {new }}(\gamma, \delta v, \theta)$ for all $\delta \in \Delta$. Similarly, we compute that

$$
\begin{aligned}
\tau^{\text {new }}(\gamma, v, \theta+1) & =\tau(\gamma \exp (-\xi) \exp (-\theta \xi), v, \theta+1) \\
& =\tau\left(\gamma \exp (-\xi) \exp (-\theta \xi) \sigma, Q^{-1} v, \theta\right) \\
& =\tau\left(\gamma \alpha \exp (-\theta \xi), Q^{-1} v, \theta\right)=\tau^{\text {new }}\left(\gamma \alpha, Q^{-1} v, \theta\right) .
\end{aligned}
$$

Since $\alpha$ has order $n$ and $Q^{2 n}=I$, it follows that $\tau^{\text {new }}(\gamma, v, \theta+2 n)=\tau^{\text {new }}(\gamma, v, \theta)$. Hence $\tau^{\text {new }}$ induces a $\Gamma$-equivariant submersion $\tau^{\text {new }}: \Gamma \times V \times S^{1} \rightarrow M$, where $S^{1}=\mathbb{R} / 2 n \mathbb{Z}$.

Since $Q=A^{-1}$ where $A$ is twisted equivariant, it follows that $Q \delta Q^{-1}=\sigma \delta \sigma^{-1}$ for $\delta \in \Delta$. Hence $\Delta$ and $Q$ generate the compact group $\Delta \rtimes \mathbb{Z}_{2 n}$ defined in the introduction. Moreover, we have a fixed-point free action of $\Delta \rtimes \mathbb{Z}_{2 n}$ on $\Gamma \times V \times S^{1}$ given by

$$
(\gamma, v, \theta) \mapsto\left(\gamma \delta^{-1}, \delta v, \theta\right), \quad \delta \in \Delta, \quad(\gamma, v, \theta) \mapsto\left(\gamma \alpha^{-1}, Q v, \theta+1\right) .
$$

It follows as in $\S 2.2$ that $\tau^{\text {new }}$ induces a $\Gamma$-equivariant diffeomorphism

$$
\tau^{\text {new }}: \frac{\left(\Gamma \times \times_{\Delta} V\right) \times S^{1}}{\mathbb{Z}_{2 n}} \cong U,
$$

where $U$ is a neighborhood of the relative periodic solution and $\Gamma \times{ }_{\Delta} V=(\Gamma \times V) / \Delta$. This completes the proof of Theorem 1.3(a). 
As before, the $\Gamma$-equivariant vector field on $U$ lifts to a $\Gamma \times\left(\Delta \rtimes \mathbb{Z}_{2 n}\right)$-equivariant vector field on $\Gamma \times V \times S^{1}$. The skew product structure (1.3) follows again from $\Gamma$-equivariance. Hence we have proved Theorem 1.3(b).

Finally, we note that the coordinates on the new bundle are related to the coordinates on the old bundle by the $\Gamma$-equivariant transformation $\gamma^{\text {new }}=\gamma \exp (\theta \xi)$. The relationship between the vector fields on the two bundles is given by

$$
f_{\Gamma}^{\text {new }}(v, \theta)=\operatorname{Ad}_{\exp (-\theta \xi)} f_{\Gamma}(v, \theta)+\xi .
$$

In particular, $f_{\Gamma}^{\text {new }}(0, \theta)=\xi$ for all $\theta \in \mathbb{R}$. To verify equation (2.7), we compute that

$$
\begin{aligned}
\dot{\gamma}^{\text {new }} & =\dot{\gamma} \exp (\theta \xi)+\gamma \exp (\theta \xi) \dot{\theta} \xi=\gamma\left\{f_{\Gamma}(v, \theta) \exp (\theta \xi)+\exp (\theta \xi) \xi\right\} \\
& =\gamma^{\text {new }}\left\{\exp (-\theta \xi) f_{\Gamma}(v, \theta) \exp (\theta \xi)+\xi\right\}=\gamma^{\text {new }} f_{\Gamma}^{\text {new }} .
\end{aligned}
$$

Remark 2.5. The $2 n$-periodicity in Theorem 1.3 holds in complete generality and depends only on the group-theoretic integer $n$ in Proposition 1.2. However, the methods of the present subsection often lead to an $n$-periodic bundle. This is the case when $Q$ can be chosen in Lemma 2.2 so that the order of $Q$ divides $n$ (for example, if $n / k$ is even).

\section{Bifurcations}

In this section, we give examples illustrating how to study bifurcation from relative periodic solutions. We assume that $\Gamma$ is an algebraic group, so that we are in the situation of Theorem 1.3. (In $\S 6$, we discuss the case when $\Gamma$ is not algebraic.)

As described in the introduction, the first step is to study bifurcation from isolated periodic solutions in the $(v, \theta)$-subsystem on $V \times S^{1}$, where $S^{1}=\mathbb{R} / 2 n \mathbb{Z}$. The $(v, \theta)$ subsystem is $\Delta \rtimes \mathbb{Z}_{2 k}$-equivariant, where the group $\Delta \rtimes \mathbb{Z}_{2 k}$ is generated by the spatial symmetries $\Delta$ of the underlying relative periodic solution $\mathcal{P}$ together with an element $Q$ of order $2 k$. Note that $\mathcal{P}$ reduces to a periodic solution $\{0\} \times S^{1} \subset V \times S^{1}$ with spatial symmetry $\Delta$ and spatiotemporal symmetry $\Delta \rtimes \mathbb{Z}_{2 k}$.

In principle, it is possible to apply the results of $[\mathbf{2 1}]$ to the $(v, \theta)$-subsystem. In practice, this is slightly confusing due to the presence of additional structure in the $(v, \theta)$-subsystem. For example, the phase space $V \times S^{1}$ is a trivial bundle over the periodic solution which was not assumed in [21]. Moreover, an important construction in [21] is a group $\Delta \rtimes \mathbb{Z}_{2 k}$ which coincides here (but not in [21]) with the spatiotemporal symmetry in the $(v, \theta)$-subsystem. For these reasons, we repeat certain calculations from [21] in the following exposition.

Some notational confusion arises from the fact that $\Delta \rtimes \mathbb{Z}_{2 k}$ acts both on $V \times S^{1}$ and on the cross-section $V \cong V \times\{0\} \subset V \times S^{1}$. To avoid confusion, we now use $Q$ to denote the action of $Q$ on $V \times S^{1}$ and $Q_{V}$ to denote the action of $Q$ on $V$. Thus $Q \cdot(v, \theta)=\left(Q_{V} v, \theta+1\right)$. (Note that $Q$ here corresponds to $\sigma$ in [21] and that there is no analogue of $Q_{V}$ in [21].)

Following the notation of [21] (with $\sigma$ replaced by $Q$ ), we let $g^{(1)}: V \rightarrow Q V$ be the first hit map for the flow in the $(v, \theta)$-subsystem. (Alternatively, we could write $g^{(1)}: V \times\{0\} \rightarrow V \times\{1\}$, where $V \times\{0\}, V \times\{1\} \subset V \times S^{1}$ are successive crosssections along the periodic solution.) Bifurcations in the $(v, \theta)$-subsystem are governed by eigenvalues of the twisted equivariant linear map $Q^{-1}\left(\mathrm{D}^{(1)}\right)_{0}: V \rightarrow V$ (cf. $L=$ 
$\sigma^{-1}\left(\mathrm{D} g^{(1)}\right)_{0}$ in [21]). In fact, this linear map coincides with the twisted equivariant linear map $L=\left.P_{0} \sigma^{-1}\left(\mathrm{D} \Phi_{1}\right)_{u_{0}}\right|_{V}$ from $\S 2.2$ :

Proposition 3.1. $L=Q^{-1}\left(\mathrm{D} g^{(1)}\right)_{0}$.

Proof. Write the time-one map $\Phi_{1}:\{\mathrm{id}\} \times V \times\{0\} \rightarrow\{\mathrm{id}\} \times V \times\{1\}$ in the $(\gamma, v, \theta)$ coordinates

$$
\Phi_{1}(\mathrm{id}, v, 0)=\left(\Phi_{1}^{\gamma}(\mathrm{id}, v, 0), \Phi_{1}^{v}(\mathrm{id}, v, 0), 1\right) .
$$

Using the identification $\left(\gamma \sigma^{-1}, v, \theta+1\right) \sim\left(\gamma, Q_{V}^{-1} v, \theta\right)$, we compute that

$$
\begin{aligned}
\sigma^{-1} \Phi_{1}(\mathrm{id}, v, 0) & =\left(\sigma^{-1} \Phi_{1}^{\gamma}(\mathrm{id}, v, 0), \Phi_{1}^{v}(\mathrm{id}, v, 0), 1\right), \\
& =\left(\operatorname{Ad}_{\sigma^{-1}} \Phi_{1}^{\gamma}(\mathrm{id}, v, 0) \sigma^{-1}, \Phi_{1}^{v}(\mathrm{id}, v, 0), 1\right) \\
& =\left(\operatorname{Ad}_{\sigma^{-1}} \Phi_{1}^{\gamma}(\mathrm{id}, v, 0), Q_{V}^{-1} \Phi_{1}^{v}(\mathrm{id}, v, 0), 0\right)
\end{aligned}
$$

It follows that

$$
L=\left.P_{0} \sigma^{-1}\left(\mathrm{D} \Phi_{1}\right)_{(\mathrm{id}, 0,0)}\right|_{V}=Q_{V}^{-1}\left(\mathrm{D}_{v} \Phi_{1}^{v}\right)_{(\mathrm{id}, 0,0)} .
$$

On the other hand,

$$
Q^{-1} g^{(1)}(v, 0)=Q^{-1}\left(\Phi_{1}^{v}(\mathrm{id}, v, 0), 1\right)=\left(Q_{V}^{-1} \Phi_{1}^{v}(\mathrm{id}, v, 0), 0\right)
$$

so that $Q^{-1}\left(\mathrm{D}^{(1)}\right)_{0,0}=Q_{V}^{-1}\left(\mathrm{D}_{v} \Phi_{1}^{v}\right)_{(\mathrm{id}, 0,0)}=L$.

By center manifold reduction, we may suppose without loss that $V$ is the center subspace of $L$. Define $\Delta_{L}$ to be the closed group generated by the actions of $\Delta$ and $L$ on $V$.

TheOREM 3.2. [21, Theorem 3.4] Suppose that the periodic solution in the $(v, \theta)$ subsystem undergoes bifurcation. Generically, the center subspace $V$ of $L$ is an irreducible representation of $\Delta_{L}$. Moreover, either $V$ is absolutely irreducible (non-Hopf bifurcation), or $V$ is irreducible of complex type (Hopf bifurcation).

We now concentrate attention on the non-Hopf case. The Hopf case is completely analogous.

Proposition 3.3. Suppose that $\Delta_{L}$ acts absolutely irreducibly on $V$. Then $L=Q_{V}^{-1}$ and $\Delta_{L} \cong \Delta \rtimes \mathbb{Z}_{2 k}$.

Proof. Observe that $L$ and $Q_{V}^{-1}$ are twisted equivariant so that $Q_{V} L: V \rightarrow V$ is an equivariant linear map. Therefore, $Q_{V} L=\alpha I$ where $\alpha \in \mathbb{R}$. It follows that $Q_{V}$ and $L$ commute and hence $\alpha^{2 k} I=\left(Q_{V} L\right)^{2 k}=Q_{V}^{2 k} L^{2 k}=L^{2 k}$. Since $V$ is the center subspace of $L$, it follows that $\alpha= \pm 1$. Finally, $Q L=\left(\mathrm{D}^{(1)}\right)_{0}$ which is equivariantly isotopic (by the flow) to the identity on $V$, ruling out the possibility that $\alpha=-1$.

Next, we define a $\Delta$-equivariant diffeomorphism $h: V \rightarrow V$ by writing $g^{(1)}(v, 0)=$ $(h(v), 1)$.

PROPOSITION 3.4. [21, Lemma 4.4] Up to arbitrarily high order, coordinates can be chosen so that $h$ is $\Delta_{L}$-equivariant. Moreover, $h$ can be regarded, to arbitrarily high order, as a general $\Delta_{L}$-equivariant diffeomorphism satisfying $h(0)=0$ and $(\mathrm{D} h)_{0}=I$. 
The analysis of bifurcation from relative periodic solutions thus divides into five steps:

(1) enumerate the absolutely irreducible representations of $\Delta_{L} \cong \Delta \rtimes \mathbb{Z}_{2 k}$;

(2) study the bifurcations of a $\Delta_{L}$-equivariant diffeomorphism $h: V \rightarrow V$ satisfying $h(0)=0$ and $(d h)_{0}=I$;

(3) interpret the results for bifurcation from the isolated periodic solution in the $(v, \theta)$ subsystem;

(4) substitute the solutions $(v(t), \theta(t))$ from Step 3 into the $\dot{\gamma}$-equation in (1.3) and solve for $\gamma(t)$;

(5) interpret the results for bifurcation from the relative periodic solution $\mathcal{P}$ in the original ODE (1.1).

We note that Steps 1 and 3 can be carried out using [21, 23]. Step 2 is covered in [6]. In this way, we may analyze the $(v, \theta)$-subsystem modulo flat terms (that are not $L$ equivariant) in the diffeomorphism $h$. It follows from determinacy results of Field [13] that many important features of the $(v, \theta)$-subsystem are unaffected by the flat terms.

Step 4 is routine in the examples considered in this paper, but is non-trivial in general. (A similar issue arises in bifurcation from relative equilibria and is made tractable there by ideas of Fiedler and Turaev [10].)

The interpretation in Step 5 is implicit in the proof of Theorem 1.3. Note that, in particular, it is necessary to pass back from the comoving frame to the original 'laboratory' frame. By concentrating on specific aspects of the bifurcation theory for $h$ in Step 2, we can state a general result about solutions bifurcating from $\mathcal{P}$ in Step 5. Recall that an isotropy subgroup $J \subset \Delta \rtimes \mathbb{Z}_{2 k}$ is called axial if the fixed-point subspace of $J$ is one dimensional.

PROPOSITION 3.5. Let $\mathcal{P}$ be a relative periodic solution for the $\Gamma$-equivariant $O D E(1.1)$ on $M$, with spatial symmetry $\Delta$ and spatiotemporal symmetry $\Sigma$ generated by $\Delta$ and $\sigma$.

Suppose that there is a non-degenerate non-Hopf bifurcation in the $\Delta \rtimes \mathbb{Z}_{2 k}$-equivariant subsystem. In particular, $\Delta_{L} \cong \Delta \rtimes \mathbb{Z}_{2 k}$ acts absolutely irreducibly on $E^{c}$. Suppose that $J$ is an axial isotropy subgroup of $\Delta_{L}$ and let $p \geq 1$ be least such that $L^{-p} \delta \in J$ for some $\delta \in \Delta$.

Then there is a branch of relative periodic solutions $\mathcal{P}^{\text {bif }}$ for the ODE (1.1) on $M$ with relative period $p$, spatial symmetry $\Delta^{\text {bif }}=J \cap \Delta$, and spatiotemporal symmetry $\Sigma^{\text {bif }}$ generated by $\Delta^{\text {bif }}$ and $\sigma^{\text {bif }}$ where $\sigma^{\text {bif }} \in Z\left(\Delta^{\text {bif }}\right) \sigma^{p} \delta$ is close to $\sigma^{p} \delta$.

Proof. Since $J$ is axial, it follows from the equivariant branching lemma $[6,15]$ that there is a branch of fixed points with isotropy $J$ for the diffeomorphism $h$. By [21, Lemma 4.7], there is a periodic solution $y(t)$ for the $(v, \theta)$-subsystem with spatial symmetry $J \cap \Delta$ and satisfying $y(p)=Q^{p} \delta y(0)$, where $p \geq 1, \delta \in \Delta$, and $p$ is least with this property.

Write $u(t)=\tau^{\text {new }}(\gamma(t), y(t))=\tau^{\text {new }}(\gamma(t), v(t), t)$, where $\tau^{\text {new }}$ is the submersion defined in $\$ 2.3$. In particular, $u(0)=\tau^{\text {new }}\left(\mathrm{id}, v_{0}, 0\right)$. The spatial symmetry of $u(t)$ is a subgroup of $\Delta$ and is independent of $t$, so it suffices to compare the isotropy subgroups of $u(0)$ and $v_{0}$. Let $\delta \in \Delta$. Then

$$
\delta u(0)=\delta \tau^{\text {new }}\left(\mathrm{id}, v_{0}, 0\right)=\tau^{\text {new }}\left(\delta, v_{0}, 0\right)=\tau^{\text {new }}\left(\mathrm{id}, \delta v_{0}, 0\right) .
$$

It follows that $\delta u(0)=u(0)$ if and only if $\delta v_{0}=v_{0}$ and hence the spatial symmetry of the 
solution $u(t)$ is given by $J \cap \Delta$. Furthermore,

$$
\begin{aligned}
u(p) & =\tau^{\text {new }}(\gamma(p), y(p))=\tau^{\text {new }}\left(\gamma(p), Q^{p} \delta y(0)\right)=\tau^{\text {new }}\left(\gamma(p), Q_{V}^{p} \delta v(0), p\right) \\
& =\tau^{\text {new }}\left(\gamma(p) \alpha^{p} \delta, v(0), 0\right)=\gamma(p) \alpha^{p} \delta u(0) .
\end{aligned}
$$

Hence $u(t)$ is a relative periodic solution with relative period $p$ (easily verified to be minimal) and spatiotemporal symmetry $\Sigma^{\text {bif }}$ generated by $\Delta^{\text {bif }}=J \cap \Delta$ and $\sigma^{\text {bif }}=$ $\gamma(p) \alpha^{p} \delta$.

Now, $\gamma(t)$ is the solution to the $\dot{\gamma}$ equation in the skew product equations (1.3) with initial condition $\gamma(0)=$ id. The solution $y(t)$ depends smoothly at least on $\sqrt{\lambda}$ (where $\lambda$ is the bifurcation parameter), so that solutions $\gamma(t, \lambda)$ to the equation $\dot{\gamma}=\gamma f_{\Gamma}(y(t, \lambda), t, \lambda)$ depend smoothly on $\sqrt{\lambda}$. Since $f_{\Gamma}(0, t, 0) \equiv \xi$, it follows that $\gamma(t, 0)=\exp t \xi$ and hence $\sigma^{\text {bif }}(\lambda)=\gamma(p, \lambda) \alpha^{p} \delta$ is close to $(\exp p \xi) \alpha^{p} \delta=\sigma^{p} \delta$.

The condition $f_{\Gamma}(\delta v, \theta)=\operatorname{Ad}_{\delta} f_{\Gamma}(v, \theta)$ implies that $f_{\Gamma}(y(t, \lambda), t, \lambda) \in \mathrm{L} Z\left(\Delta^{\text {bif }}\right)$ for all $t, \lambda \in \mathbb{R}$. Hence $\gamma(t, \lambda) \in Z\left(\Delta^{\text {bif }}\right)$. It follows that $\sigma^{\text {bif }} \in Z\left(\Delta^{\text {bif }}\right) \sigma^{p} \delta$.

Remark 3.6. In Proposition 3.5, we have concentrated attention on the existence of relative periodic solutions arising from axial isotropy subgroups $J \subset \Delta_{L}=\Delta \rtimes \mathbb{Z}_{2 k}$. More detailed bifurcation results (such as the existence of relative periodic solutions corresponding to non-axial isotropy subgroup $J$, stabilities, and so on) follow similarly from a more detailed analysis of the associated bifurcation for the $\Delta_{L}$-equivariant diffeomorphism $h$.

In the remainder of this section, we consider examples of non-Hopf bifurcation from modulated rotating waves (Example 3.7) and modulated traveling waves (Example 3.8).

Example 3.7. (Modulated rotating wave) A modulated rotating wave is a relative periodic solution that is periodic in a corotating frame. We consider modulated rotating waves with spatiotemporal symmetry $\Sigma=\mathbf{S O}(2)$ and $\Delta=\mathbb{Z}_{\ell}(\ell \geq 1)$ in systems with symmetry (a) $\Gamma=\mathbf{S O}(2)$ and (b) $\Gamma=\mathbf{E}(2)$. Such modulated rotating waves arise in a variety of different forms in cellular flame experiments $[3,16]$ (with $\Gamma=\mathbf{S O}(2)$ ), and as 'meandering $(\ell$-armed) spiral waves' in chemical reactions [32] (with $\Gamma=\mathbf{S E}(2)$ ).

(a) $\Gamma=\mathbf{S O}(2)$. It is immediate that $k=n=1$. Moreover, the element $\sigma$ (which is generically an irrational rotation) can be written as $\sigma=\exp \xi$ for some $\xi \in \operatorname{LSO}(2)$. In particular, $\alpha=$ id.

Passing to the skew product equations (1.3) in the corotating frame rotating with speed $\xi$, the modulated rotating wave becomes an ordinary periodic solution with spatial symmetry $\Delta=\mathbb{Z}_{\ell}$ and no further spatiotemporal symmetry.

Since $\Delta \rtimes \mathbb{Z}_{2 k}=\mathbb{Z}_{\ell} \times \mathbb{Z}_{2}$ is abelian, the absolutely irreducible representations $V$ are one dimensional. Let $V_{+}$denote the trivial irreducible representation of $\Delta=\mathbb{Z}_{\ell}$. When $\ell$ is even, there is also a non-trivial irreducible representation $V_{-}$with kernel $\mathbb{Z}_{\ell / 2}$. Since $\operatorname{dim} V=1$, there is a unique axial isotropy subgroup $J \subset \Delta \rtimes \mathbb{Z}_{2 k}$, namely the subgroup of $\Delta \rtimes \mathbb{Z}_{2 k}$ that acts trivially on $V$. Applying Proposition 3.5, it is now straightforward to verify the entries in Table 1 .

The notion of period preserving/doubling depends a priori on the choice of comoving frame. However, in this example, there is a natural choice of $\sigma$, namely $\sigma=\exp \xi$ with 
TABLE 1. Spatiotemporal symmetry of bifurcating solutions in non-Hopf bifurcation from a modulated rotating wave with $\Gamma=\mathbf{O}(2), \Sigma=\mathbf{S O}(2), \Delta=\mathbb{Z}_{\ell}, \ell \geq 1$. All bifurcations are to modulated rotating waves. All bifurcations are period preserving pitchforks bifurcations unless stated otherwise. Period preserving and period doubling refers to periodicity in the corotating frame (ignoring new slow drifts). $R_{\pi / \ell}$ is the generator of $\mathbb{Z}_{\ell}$.

\begin{tabular}{lrccccccl}
\hline Space & $L$ & $J$ & $\Delta^{\text {bif }}$ & $p$ & $\alpha^{p} \delta$ & $Z\left(\Delta^{\text {bif }}\right)^{0}$ & $\Sigma^{\text {bif }}$ & Remarks \\
\hline$V_{+}$ & 1 & $\left\langle R_{2 \pi / \ell}, L\right\rangle$ & $\mathbb{Z}_{\ell}$ & 1 & id & $\mathbf{S O}(2)$ & $\mathbf{S O}(2)$ & Saddle-node \\
$V_{+}$ & -1 & $\left\langle R_{2 \pi / \ell}\right\rangle$ & $\mathbb{Z}_{\ell}$ & 2 & id & $\mathbf{S O}(2)$ & $\mathbf{S O}(2)$ & Period doubling \\
$V_{-}(\ell$ even $)$ & 1 & $\left\langle R_{4 \pi / \ell}, L\right\rangle$ & $\mathbb{Z}_{\ell / 2}$ & 1 & id & $\mathbf{S O}(2)$ & $\mathbf{S O}(2)$ & \\
$V_{-}(\ell$ even $)$ & -1 & $\left\langle L R_{2 \pi / \ell}\right\rangle$ & $\mathbb{Z}_{\ell / 2}$ & 1 & $R_{2 \pi / \ell}$ & $\mathbf{S O}(2)$ & $\mathbf{S O}(2)$ & Period doubling \\
\hline
\end{tabular}

TABLE 2. Spatiotemporal symmetry of bifurcating solutions in non-Hopf bifurcation from a modulated traveling wave with $\Gamma=\mathbf{S E}(2), \Sigma=\mathbb{D}_{1} \times \mathbb{Z}, \Delta=\mathbb{D}_{1}$. The entries for $\Sigma^{\text {bif }}$ are given only up to conjugacy. All bifurcations are period preserving pitchforks of modulated traveling waves unless stated otherwise.

\begin{tabular}{lrccccccl}
\hline Space & $L$ & $J$ & $\Delta^{\text {bif }}$ & $p$ & $\alpha^{p} \delta$ & $Z\left(\Delta^{\text {bif }}\right)^{0}$ & $\Sigma^{\text {bif }}$ & Remarks \\
\hline$V_{+}$ & 1 & $\langle\kappa, L\rangle$ & $\mathbb{D}_{1}$ & 1 & id & $\mathbb{R}$ & $\mathbb{D}_{1} \times \mathbb{Z}$ & Saddle-node \\
$V_{+}$ & -1 & $\langle\kappa\rangle$ & $\mathbb{D}_{1}$ & 2 & id & $\mathbb{R}$ & $\mathbb{D}_{1} \times \mathbb{Z}$ & Period doubling \\
$V_{-}$ & 1 & $\langle L\rangle$ & $\mathbf{1}$ & 1 & id & $\mathbf{S E}(2)$ & $\mathbf{S O}(2)$ & Modulated rotating wave \\
$V_{-}$ & -1 & $\langle L \kappa\rangle$ & $\mathbf{1}$ & 1 & $\kappa$ & $\mathbf{S E}(2)$ & $\mathbb{Z}$ & Period doubling \\
\hline
\end{tabular}

$\alpha=$ id. In the corresponding corotating frame, the underlying modulated rotating wave reduces to a periodic solution with a well-defined absolute period (in this case, absolute period 1).

Similarly, the bifurcating relative periodic solutions have well-defined absolute periods in the corotating frame modulo new slow drifts on $Z\left(\Delta^{\text {bif }}\right)^{0}$. Define $q \geq 1$ to be the least integer such that $\delta^{q}=\left(\alpha^{p} \delta\right)^{q} \in \Delta^{\text {bif }}$. Then the absolute period modulo slow drifts is approximately $p q$ and depends only on the choice of $\sigma$. Since we have a natural choice of $\sigma$ in this example (and also in Example 3.8 below), we may speak of period preserving and period doubling bifurcations.

We caution that even with the natural choice of $\sigma$, we are not claiming that there is a 'natural' comoving frame (since the equation $\exp \xi=\sigma$ does not determine $\xi$ uniquely).

(b) $\Gamma=\mathbf{S E}(2)$. This is almost identical to the case $\Gamma=\mathbf{O}(2)$. In particular, we have $k=n=1, \alpha=\mathrm{id}, \Delta \rtimes \mathbb{Z}_{2 k}=\mathbb{Z}_{\ell} \times \mathbb{Z}_{2}$ as before. The entries in Table 1 are unchanged except that when $\Delta^{\text {bif }}=1$ we obtain $Z\left(\Delta^{\text {bif }}\right)^{0}=\mathbf{S E}(2)$. This change occurs only when $\ell=1$ and in the $V_{-}$cases when $\ell=2$.

Under the assumption (valid generically) that $\xi \neq 0$, the only change in the conclusions when $Z\left(\Delta^{\text {bif }}\right)^{0}=\mathbf{S E}(2)$ is that the center of approximate rotation of the bifurcating modulated rotating waves varies periodically in time (cf. [14]).

Example 3.8. (Modulated traveling wave) A modulated traveling wave is a relative periodic solution that is periodic in a cotraveling frame. We consider non-Hopf bifurcation 
from a modulated traveling wave with $\Gamma=\mathbf{S E}(2), \Sigma=\mathbb{D}_{1} \times \mathbb{Z}$ and $\Delta=\mathbb{D}_{1}$. Such modulated traveling waves arise for example as 'bound pairs of spiral waves'; see [8].

Observe that $Z(\Sigma)^{0} \cong \mathbb{R}$. It is immediate that $k=n=1$. Moreover, the element $\sigma$ (which is generically a translation) can be written as $\sigma=\exp \xi$ for some $\xi \in \mathrm{L} \mathbb{R}$. In particular, $\alpha=$ id. Again, we pass to the skew product equations (1.3) in the cotraveling frame traveling with speed $\xi$. Our results are given in Table 2.

\section{Relative periodic solutions and comoving frames}

In this section we study the structure of the relative periodic solution itself.

In $\S 4.1$, we recall some basic results concerning topologically cyclic subgroups and Cartan subgroups. As shown in $[\mathbf{2 , ~ 1 2 , ~ 2 0 ] , ~ t h e s e ~ c o n c e p t s ~ d r i v e ~ t h e ~ d y n a m i c s ~ o n ~ r e l a t i v e ~}$ periodic solutions. These results are described and extended slightly in $\S 4.2$.

In $\S 4.3$, we introduce the index $m$ of a relative periodic solution. Provided $\Gamma$ is algebraic, the index $m$ is finite and divides the integer $n$ in Proposition 1.2. Moreover, we show that $m$ is stable if $\Gamma$ is compact and also if $\Gamma$ is the Euclidean group $\mathbf{E}(N)$ for some $N$.

In $\S \S 4.4$ and 4.5 , we consider relative periodic solutions with finite index $m$. In $\S 4.4$, we show that the relative periodic solution reduces to a group orbit of periodic solutions of period $m$ in a suitable moving frame. In $\S 4.5$, we show that a neighborhood of the relative periodic solution can be written as a $2 m$-periodic bundle. This is the optimal periodicity that may be obtained in general.

4.1. Topologically cyclic subgroups and Cartan subgroups. We begin by recalling the main definitions and results concerning Cartan subgroups [4]. Let $G$ be a finitedimensional Lie group. We denote the connected component of the identity by $G^{0}$, and define the projection $\pi: G \rightarrow G / G^{0}$. Let $g \in G$ and define $H(g)$ to be the closure of the subgroup of $G$ generated by $g$. Such a subgroup $H(g)$ is said to be topologically cyclic and either $H(g) \cong \mathbb{Z}$ or $H(g) \cong T^{p} \times \mathbb{Z}_{q}$ where $p \geq 0, q \geq 1$ are integers. Note that $\pi(H(g))=\langle\pi(g)\rangle$, where $\langle\pi(g)\rangle \subset G / G^{0}$ is the cyclic subgroup generated by $\pi(g)$.

We concentrate on the case when $H(g)$ is compact. In particular, the cyclic group $\pi(H(g))$ is finite. Define $\widetilde{G}=\pi^{-1} \pi(H(g))$. Then $\widetilde{G}$ is a finite-dimensional Lie group consisting of finitely many connected components of $G$. Hence $\widetilde{G}$ is diffeomorphic to $K \times \mathbb{R}^{a}$ where $a \geq 0$ and $K$ is the (unique up to conjugacy) maximal compact subgroup of $\widetilde{G}$; see for example [5]. Observe that $\langle\pi(g)\rangle=\pi(H(g))=\pi(K)=\pi(\widetilde{G})$. It follows from the theory of Cartan subgroups [4] that there is a maximal (with respect to inclusion) topologically cyclic subgroup $H \subset K$ containing $g$ such that $\pi(H)=\langle\pi(g)\rangle$. This subgroup $H$ is called the Cartan subgroup corresponding to $g$ and is unique up to conjugacy. In fact, it is sufficient to require maximality with respect to dimension in defining $H$ (instead of maximality with respect to inclusion).

We note that this construction depends only on $\pi(g)$. More precisely, if $g_{1}$ and $g_{2}$ lie in the same connected component of $G$, and $H\left(g_{1}\right)$ and $H\left(g_{2}\right)$ are compact, then (up to conjugacy) we obtain the same group $\widetilde{G} \cong K \times \mathbb{R}^{a}$ and the same Cartan subgroup $H$ corresponding to $g_{1}$ and $g_{2}$. Moreover, for generic $g$ in this connected component of $G$, either $H(g) \cong \mathbb{Z}$ or $H(g) \cong H[4]$. 
Definition 4.1. Let $g \in G$ and suppose that $H(g)$ is compact. Let $H$ be the Cartan subgroup corresponding to $g$ and write $H \cong T^{d} \times \mathbb{Z}_{m}$. We say that $g$ has rank $d$ and index $m$.

Example 4.2. Let $G$ be the non-split extension of $\mathbf{S O}(2)$ by an element $g$ such that $g^{2}=R_{\pi} \in \mathbf{S O}(2)$. A matrix representation of this group is

$$
R_{\theta}=\left(\begin{array}{cccc}
\cos \theta & -\sin \theta & 0 & 0 \\
\sin \theta & \cos \theta & 0 & 0 \\
0 & 0 & \cos \theta & \sin \theta \\
0 & 0 & -\sin \theta & \cos \theta
\end{array}\right), \quad g=\left(\begin{array}{cccc}
0 & 0 & 1 & 0 \\
0 & 0 & 0 & 1 \\
-1 & 0 & 0 & 0 \\
0 & -1 & 0 & 0
\end{array}\right) .
$$

The Cartan subgroup corresponding to $g$ is isomorphic to $\mathbb{Z}_{4}$, although $G=\widetilde{G}$ has only two connected components.

Thus, even when $G$ is compact, the index $m$ of an element $g \in G$ may be larger than the number of connected components of $G$ in contrast to what is claimed in [12, Lemmas 4.1,4.2], [2, Theorem 4.1, Proposition 4.3]. (This error is of no consequence for the results in $[2,12]$.)

4.2. Dynamics on relative periodic solutions. We now return to the set up of vector fields with relative periodic solutions. Let $\Gamma$ be a finite-dimensional Lie group acting smoothly and properly on a finite-dimensional manifold $M$. Let $\mathcal{F}: M \rightarrow T M$ be a smooth $\Gamma$-equivariant vector field with flow $\Phi_{t}$, and suppose that $\mathcal{P}$ is a relative periodic solution with relative period 1 . Choose $u_{0} \in \mathcal{P}$ and write $\Phi_{1}\left(u_{0}\right)=\sigma u_{0}$ where $\sigma \in \Gamma$.

As usual, we have the (compact) spatial symmetry group $\Delta$ and the spatiotemporal symmetry group $\Sigma$. Recall that $\sigma \in N(\Delta)$ and that $\Sigma$ is the closed subgroup of $N(\Delta)$ generated by $\Delta$ and $\sigma$. Observe that $\Sigma / \Delta$ is the topologically cyclic subgroup of $N(\Delta) / \Delta$ generated by the coset $\sigma \Delta$.

Now, $\Sigma$ is compact if and only if $\Sigma / \Delta$ is compact, in which case $\operatorname{dim} \Sigma / \Delta \leq d$ where $d$ is the rank of $\sigma \Delta \in N(\Delta) / \Delta$. Moreover, when $\Sigma$ is compact, it is generically the case that $\operatorname{dim} \Sigma / \Delta=d$; see $[\mathbf{2}, \mathbf{1 2}, \mathbf{2 0}]$. (Here, genericity is within the class of smooth $\Gamma$-equivariant vector fields $\mathcal{F}: M \rightarrow T M$ with relative periodic solution $\mathcal{P}$.)

Altogether, we have the following result $[\mathbf{2}, \mathbf{1 2}, \mathbf{2 0}]$. (In contrast to [2], we do not require that $N(\Delta) / \Delta$ has finitely many connected components.)

Proposition 4.3. Let $\Gamma$ be a finite-dimensional Lie group, and let $\mathcal{P}$ be a relative periodic solution. If $\Sigma$ is non-compact, then $\mathcal{P}$ is foliated by unbounded trajectories. If $\Sigma$ is compact, then $\mathcal{P}$ is foliated by $(p+1)$-dimensional tori with $(p+1)$-frequency quasiperiodic flows for some $p \leq d$, where $d$ is the rank of $\sigma \Delta \in N(\Delta) / \Delta$. Moreover, generically $p=d$.

4.3. The index of a relative periodic solution. We assume the set up of $\S 4.2$.

Definition 4.4. Suppose that $\Gamma$ is a finite-dimensional Lie group and that $\mathcal{P}$ is a relative periodic solution. Then the index of $\mathcal{P}$ is the least positive integer $m$ such that $\sigma^{m} \in$ $\exp \operatorname{L}(\Sigma) \cdot \Delta$. If no such integer $m$ exists, we say that $\mathcal{P}$ has infinite index. 
In the remainder of this subsection, we show how the index $m$ in Definition 4.4 is related to the integer $m$ in Definition 4.1 and to the integer $n$ in Proposition 1.2.

PROPOSITION 4.5. Suppose that $\Gamma$ is a compact Lie group and that $\mathcal{P}$ is a relative periodic solution. Then the index of $\mathcal{P}$ is finite and coincides with the index of the element $\sigma \Delta \in N(\Delta) / \Delta$.

Proof. Without loss, we may suppose that $\Delta=1$. In particular, $Z(\Sigma)=Z(\sigma)$.

Let $m$ be the index of $\mathcal{P}$ and let $q$ be the index of $\sigma$, so that the Cartan subgroup corresponding to $\sigma$ has the form $H=T^{d} \times \mathbb{Z}_{q}$. We show that $q=m$.

Since $\sigma \in H=T^{d} \times \mathbb{Z}_{q}$, it follows that $\sigma^{q} \in T^{d} \subset Z(\sigma)^{0}$ and hence $m$ divides $q$. In particular, $m$ is finite.

It remains to show that $q$ is the least such positive integer. Since $\sigma^{m} \in T^{d}$, we can form a subgroup $\widehat{H}=T^{d} \times \mathbb{Z}_{m} \subset H$ generated by $T^{d}$ and $\sigma$. But $\widehat{H}$ is a topologically cyclic subgroup containing $\sigma$ and satisfying $\pi(\widehat{H})=\langle\pi(\sigma)\rangle$. Since $\widehat{H}$ has the same (hence maximal) dimension as $H$ it follows that $\widehat{H}$ is the Cartan subgroup corresponding to $\sigma$. Hence $q=m$.

COROLlary 4.6. Suppose that $\Gamma$ is a compact Lie group. Then the index $m$ of a relative periodic solution is stable under perturbations (of the element $\sigma \in N(\Delta)$ ).

Proof. By Proposition 4.5, the index $m$ is determined by the Cartan subgroup $H$ corresponding to $\sigma \Delta$. But $H$ depends only on the connected component of $N(\Delta) / \Delta$ containing $\sigma \Delta$ and hence is stable under perturbations.

COROLlaRY 4.7. Let $\Gamma=G \ltimes \mathbb{R}^{N}$ be the semidirect product of a compact subgroup $G \subset \mathbf{O}(N)$ and a normal vector subgroup $\mathbb{R}^{N}, N \geq 1$. Suppose that $\Delta=1$. Then the index $m$ of a relative periodic solution is finite and stable under perturbations (of the element $\sigma \in \Gamma$ ).

Proof. Write $\sigma \in \Gamma$ in the form $\sigma=(R, w)$, where $R \in G, w \in \mathbb{R}^{N}$. We show that the index $m$ of $\sigma$ in $\Gamma$ coincides with the index $m_{R}$ of $R \in G$. The result then follows immediately from Corollary 4.6.

First, we verify that $m_{R} \leq m$. Suppose that $\sigma^{m}=\exp \xi$ where $\xi \in \mathrm{L} Z(\sigma)$, and write $\xi=\left(\xi_{R}, \xi_{w}\right)$ where $\xi_{R} \in \mathrm{L} G, \xi_{w} \in \mathrm{LR}^{N}$. It is an immediate consequence of the semidirect product structure that $R^{m}=\exp \xi_{R}$ and $\xi_{R} \in \mathrm{L} Z(R)$. Hence $R$ has index $m_{R} \leq m$.

Next, we verify that $m \leq m_{R}$. Conjugating $\sigma=(R, w)$ by an element of the form $(I, y)$ where $y \in \mathbb{R}^{N}$ is chosen appropriately, we can transform $\sigma$ into an element $\sigma=(R, w)$ where $R w=w$. Since $G$ is compact, $R$ has finite index $m_{R}$. Hence there is an element $\xi_{R} \in \mathrm{L} G$ such that $R^{m_{R}}=\exp \xi_{R}$ and $\operatorname{Ad}_{R} \xi_{R}=\xi_{R}$. Let $\xi=\left(\xi_{R}, m_{R} w\right) \in \mathrm{L} \Gamma$. A calculation shows that $\sigma^{m_{R}}=\exp \xi$ and $\operatorname{Ad}_{\sigma} \xi=\xi$. Hence $\sigma$ has index $m \leq m_{R}$.

Remark 4.8. Corollaries 4.6 and 4.7 show that the index $m$ is stable for compact groups and for groups that are 'Euclidean-like' (taking $G=\mathbf{O}(N)$ in Corollary 4.7 yields the Euclidean group $\Gamma=\mathbf{E}(N))$. 
However, in general, the index $m$ need not be stable to perturbations of the element $\sigma \in N(\Delta)$. For example, consider the case $\Gamma=\mathbf{S L}(2, \mathbb{R}), \Delta=\mathbf{1}$. The image of the exponential map exp $: \mathbf{L S L}(2, \mathbb{R}) \rightarrow \mathbf{S L}(2, \mathbb{R})$ consists of those $2 \times 2$ matrices $\gamma \in \Gamma$ for which $\operatorname{tr} \gamma>-2$ together with $\gamma=-I$; see for example [5, p. 74]. If $\sigma$ lies in the image of the exponential map, then $m=1$. Otherwise, $m=2$. Hence the index $m$ of $\sigma \in \Gamma$ is stable if and only if $\operatorname{tr} \sigma \neq-2$.

PROPOSITION 4.9. The relative periodic solution $\mathcal{P}$ has finite index $m$ if and only if $\sigma$ can be chosen (within $\sigma \Delta$ ) so that $\sigma^{n} \in \exp \operatorname{LZ}(\Sigma)$ for some positive integer $n$.

In particular, if $\Gamma$ is algebraic, then $\mathcal{P}$ has finite index.

Proof. Suppose that $\sigma^{m}=\exp (\zeta) \delta_{0}$ where $\zeta \in \underset{\sim}{Z}(\Sigma)$ and $\delta_{0} \underset{\sim}{\in} \Delta$. Define the compact Lie group $\widetilde{\Delta}=\Delta \cap Z(\sigma)$ and observe that $\delta_{0} \in \widetilde{\Delta}$. Let $H \subset \widetilde{\Delta}$ be the Cartan subgroup containing $\delta_{0}$. Then $\delta_{0}^{q} \in H^{0}$ for some $q \geq 1$, and we can write $\delta_{0}^{q}=\epsilon^{m q}$ where $\epsilon \in \widetilde{\Delta}$. Let $\widetilde{\sigma}=\sigma \epsilon^{-1} \in \sigma \Delta$. Then

$$
\tilde{\sigma}^{m q}=\sigma^{m q} \epsilon^{-m q}=\exp (q \zeta) \delta_{0}^{q} \epsilon^{-m q} \in \operatorname{expLZ}(\Sigma) .
$$

Take $n=m q$. This proves the non-trivial direction in the first statement of the proposition and the second statement follows from Proposition 1.2.

When the index $m$ is finite, we have that $m$ divides the integer $n$ in Proposition 1.2. Note also that $m$ depends only on the $\operatorname{coset} \sigma \Delta$, whereas $n$ depends on the choice of $\sigma$ within this coset.

4.4. Structure of a relative periodic solution. It is clear that a relative equilibrium with spatial symmetry $\Delta$ is diffeomorphic to $\Gamma / \Delta$. Similarly, for a relative periodic solution $\mathcal{P}$, we have

$$
\mathcal{P} \cong \frac{(\Gamma / \Delta) \times \mathbb{R}}{\mathbb{Z}} \cong \frac{\Gamma \times \mathbb{R}}{\Delta \rtimes \mathbb{Z}}
$$

where the action of $\Delta \rtimes \mathbb{Z}$ on $\Gamma \times \mathbb{R}$ is given by

$$
(\gamma, \theta) \mapsto\left(\gamma \delta^{-1}, \theta\right), \quad \delta \in \Delta, \quad(\gamma, \theta) \mapsto\left(\gamma \sigma^{-1}, \theta+1\right)
$$

When $\mathcal{P}$ has finite index $m$, we can simplify this representation of $\mathcal{P}$ by passing to a suitable comoving frame. In particular, we show that in a comoving frame $\mathcal{P}$ is the quotient by a compact Lie group of an $m$-periodic trivial bundle $\Gamma \times S^{1}$. Here, $m$-periodicity is optimal.

Write $\sigma^{m}=\delta_{0} \exp m \zeta$, where $\zeta \in \mathrm{L} Z(\Sigma)$ and $\delta_{0} \in \Delta$. Let $\Sigma_{m}$ denote the subgroup of $\Gamma$ generated by $\Delta$ and $\beta=\exp (-\zeta) \sigma$. Note that $\beta \in N(\Delta)$ and that $\beta^{m} \in \Delta$. Hence $\Sigma_{m}$ is a cyclic extension of $\Delta$ of order $m$, so $\Sigma_{m} / \Delta \cong \mathbb{Z}_{m}$. We call $\Sigma_{m}$ the discrete spatiotemporal symmetry group of the relative periodic solution.

LemMa 4.10. Suppose that $\mathcal{P}$ has finite index $m$. Write $\sigma^{m}=\delta_{0} \exp m \zeta$ where $\zeta \in$ $\mathrm{LZ}(\Sigma)$ and $\delta_{0} \in \Delta$. Define $\beta$ and $\Sigma_{m}$ as above. Then, in a comoving frame moving with velocity $\zeta$, 


$$
\mathcal{P} \cong \frac{(\Gamma / \Delta) \times S^{1}}{\mathbb{Z}_{m}} \cong \frac{\Gamma \times S^{1}}{\Sigma_{m}}
$$

where $S^{1}=\mathbb{R} / m \mathbb{Z}$.

The action of $\Sigma_{m}$ on $\Gamma \times S^{1}$ is given by

$$
(\gamma, \theta) \mapsto\left(\gamma \delta^{-1}, \theta\right), \quad \delta \in \Delta, \quad(\gamma, \theta) \mapsto\left(\gamma \beta^{-1}, \theta+1\right) .
$$

Proof. Define the smooth parameterization $\tau: \Gamma \times \mathbb{R} \rightarrow \mathcal{P}$ given by

$$
\tau(\gamma, \theta)=\gamma \exp (-\zeta \theta) \Phi_{\theta}\left(u_{0}\right)
$$

Since $\zeta \in \mathrm{LZ}(\Delta)$, we have that $\tau\left(\gamma \delta^{-1}, \theta\right)=\tau(\gamma, \theta)$ for all $\delta \in \Delta$. Moreover, since $\zeta \in \mathrm{L} Z(\sigma)$, we have that $\tau(\gamma, \theta+1)=\gamma \exp (-\zeta) \exp (-\zeta \theta) \sigma \Phi_{\theta}\left(u_{0}\right)=\tau(\gamma \beta, \theta)$. In particular, $\tau(\gamma, \theta+m)=\tau\left(\gamma \beta^{m}, \theta\right)=\tau\left(\gamma \delta_{0}, \theta\right)=\tau(\gamma, \theta)$. Hence $\tau$ induces a smooth map $\tau: \Gamma \times S^{1} \rightarrow \mathcal{P}$ with the required properties.

In the comoving frame in Lemma 4.10, the relative periodic solution $\mathcal{P}$ becomes a group orbit of ordinary periodic solutions with spatial symmetry $\Delta$ and spatiotemporal symmetry $\Sigma_{m}$. Moreover, the integer $m$ is the least possible. When $\mathcal{P}$ is a discrete rotating wave, $m$ is the absolute period of $\mathcal{P}$, and $\Sigma_{m}=\Sigma$. Hence the index $m$ corresponds to the integer $m$ in [21].

4.5. A 2m-periodic bundle. We continue to suppose that $\mathcal{P}$ is a relative periodic solution with finite index $m$. In $\S 4.4$, we passed to a comoving frame in which $\mathcal{P}$ could be represented as an $m$-periodic bundle; more precisely, the quotient of an $m$-periodic trivial bundle by a free compact Lie group action. Here, the integer $m$ is the least possible. It is natural to ask whether a neighborhood of $\mathcal{P}$ can be represented as a $2 m$-periodic bundle. (A factor of two is to be expected to take account of orientability issues.) In this subsection, we answer this question positively.

Although the $2 n$-periodic bundle obtained in $\S 2$ is adequate for applications, there are at least three reasons for looking for an $2 m$-periodic bundle. First, the integer $m$ is intrinsically defined, independent of any choices. Second, $m$ is optimally small. (Recall that in general $m$ divides $n$.) Third, this bundle makes transparent how the action of the discrete spatiotemporal symmetry group $\Sigma_{m}$ on the cross-section $V$ comes about. Overall, the $2 m$-periodic bundle seems more natural.

Write $\sigma^{m}=\delta_{0} \exp m \zeta$ where $\zeta \in \mathrm{LZ}(\Sigma)$ and $\delta_{0} \in \Delta$. In $\S 4.3$, we introduced the discrete spatiotemporal symmetry group $\Sigma_{m}$, namely the subgroup of $\Gamma$ generated by $\Delta$ and $\beta=\exp (-\zeta) \sigma$.

The group $\Sigma_{m}$ is a cyclic extension of $\Delta$ of order $m$. To take account of orientability problems, we define a related cyclic extension $\Sigma_{2 m}$.

PROPOSITION 4.11. Let $\Sigma_{2 m}$ be the group generated by $\Delta$ and an element $R$ such that $R \delta R^{-1}=\sigma \delta \sigma^{-1}$, for $\delta \in \Delta$, and $R^{2 m}=\delta_{0}^{2}$. Then $\Sigma_{2 m}$ is a cyclic extension of $\Delta$ of order $2 m$. 
Proof. The group $\Sigma_{2 m}$ can be realized as a matrix group:

$$
\left(\begin{array}{ll}
\delta & 0 \\
0 & 1
\end{array}\right), \quad \delta \in \Delta, \quad\left(\begin{array}{cc}
\beta & 0 \\
0 & e^{\pi i / m}
\end{array}\right) .
$$

THEOREM 4.12. Let $\mathcal{P}$ be a relative periodic solution with finite index $m$, and write $\sigma^{m}=\delta_{0} \exp m \zeta$, where $\zeta \in \operatorname{LZ}(\Sigma)$ and $\delta_{0} \in \Delta$. Form the group $\Sigma_{2 m}$ generated by $\Delta$ and $R$ as in Proposition 4.11. Then, there is a neighborhood $U$ of the relative periodic solution $\mathcal{P}$ such that, in a comoving frame, moving uniformly with velocity $\zeta$,

$$
U \cong \frac{\left(\Gamma \times{ }_{\Delta} V\right) \times S^{1}}{\mathbb{Z}_{2 m}} \cong \frac{\Gamma \times V \times S^{1}}{\Sigma_{2 m}},
$$

where $V$ is a representation of the group $\Sigma_{2 m}$, and $S^{1}=\mathbb{R} / 2 m \mathbb{Z}$.

The action of $\Sigma_{2 m}$ on $\Gamma \times V \times S^{1}$ is given by

$$
\delta \cdot(\gamma, v, \theta)=\left(\gamma \delta^{-1}, \delta v, \theta\right), \quad \delta \in \Delta, \quad R \cdot(\gamma, v, \theta)=\left(\gamma \beta^{-1}, R v, \theta+1\right),
$$

where $\beta=\exp (-\zeta) \sigma$.

Proof. Let $A: V \rightarrow V$ be the orthogonal twisted equivariant linear map in equation (2.2). Let $G$ be the closed subgroup of $\mathbf{O}(V)$ acting on $V$ generated by the actions of $\Delta$ and $A$ and let $Z$ denote the centralizer of $G$ in $\mathbf{O}(V)$.

We claim that $\delta_{0} A^{m} \in Z$. Note that $\sigma^{-m} \delta_{0} \in Z(\Delta)$ so that $\delta_{0} \delta=\sigma^{m} \delta \sigma^{-m} \delta_{0}$, for all $\delta \in \Delta$. In other words, $\delta_{0} \delta=\phi^{-m}(\delta) \delta_{0}$, for all $\delta \in \Delta$. By twisted equivariance, $A^{m} \delta=\phi^{m}(\delta) A^{m}$ for all $\delta \in \Delta$. Hence the composition $\delta_{0} A^{m}$ commutes with elements of $\Delta$. At the same time, $A \delta_{0}=\phi\left(\delta_{0}\right) A=\delta_{0} A$ since $\delta_{0}$ commutes with $\sigma$. It follows that $\delta_{0} A^{m}$ commutes with $A$ proving the claim.

Now, $Z$ is a space of equivariant linear maps, and it follows from general arguments, see the appendix, that $B^{2} \in Z^{0}$ for all $B \in Z$. Hence $\delta_{0}^{2} A^{2 m} \in Z^{0}$. Since $Z$ is compact, we can write $\delta_{0}^{2} A^{2 m}=\exp (2 m \eta)$ where $\eta \in \mathrm{L} Z$. In particular, $\exp (t \eta)$ is $\Delta$-equivariant and commutes with $A$ for all $t \in \mathbb{R}$. Set $R=Q \exp (\eta)$, where $Q=A^{-1}$ so that $R^{2 m}=\delta_{0}^{2}$.

Let $\tau: \Gamma \times V \times \mathbb{R} \rightarrow M$ be the submersion defined in $\$ 2.2$. As in the proof of Theorem 1.3(a), we consider a modified submersion $\tau^{\text {new }}$. This time, we define

$$
\tau^{\text {new }}(\gamma, v, \theta)=\tau(\gamma \exp (-\theta \zeta), \exp (-\theta \eta) v, \theta) .
$$

As before, $\tau^{\text {new }}(\gamma \delta, v, \theta)=\tau^{\text {new }}(\gamma, \delta v, \theta)$ for all $\delta \in \Delta$, and we compute that

$$
\begin{aligned}
\tau^{\text {new }}(\gamma, v, \theta+1) & =\tau(\gamma \exp (-\zeta) \exp (-\theta \zeta), \exp (-\eta) \exp (-\theta \eta) v, \theta+1) \\
& =\tau\left(\gamma \exp (-\zeta) \exp (-\theta \zeta) \sigma, Q^{-1} \exp (-\eta) \exp (-\theta \eta) v, \theta\right) \\
& =\tau\left(\gamma \beta \exp (-\theta \zeta), \exp (-\theta \eta) R^{-1} v, \theta\right)=\tau^{\text {new }}\left(\gamma \beta, R^{-1} v, \theta\right) .
\end{aligned}
$$

Since $\beta^{m}=\delta_{0}$ and $R^{2 m}=\delta_{0}^{2}$, it follows that

$$
\tau^{\text {new }}(\gamma, v, \theta+2 m)=\tau^{\text {new }}\left(\gamma \delta_{0}^{2}, \delta_{0}^{-2} v, \theta\right)=\tau^{\text {new }}(\gamma, v, \theta)
$$

Hence $\tau^{\text {new }}$ induces a $\Gamma$-equivariant submersion $\tau^{\text {new }}: \Gamma \times V \times S^{1} \rightarrow M$, where $S^{1}=\mathbb{R} / 2 m \mathbb{Z}$. Moreover, we have a fixed-point free action of $\Sigma_{2 m}$ given by

$$
(\gamma, v, \theta) \mapsto\left(\gamma \delta^{-1}, \delta v, \theta\right), \quad \delta \in \Delta, \quad(\gamma, v, \theta) \mapsto\left(\gamma \beta^{-1}, R v, \theta+1\right) .
$$

It follows that $\tau$ induces a $\Gamma$-equivariant diffeomorphism $\tau:\left(\left(\Gamma \times{ }_{\Delta} V\right) \times S^{1}\right) / \mathbb{Z}_{2 m} \cong U$, where $U$ is a neighborhood of $\mathcal{P}$ and $\Gamma \times{ }_{\Delta} V=(\Gamma \times V) / \Delta$. 
Remark 4.13. (a) As was the case for the $2 n$-periodic bundle in Theorem 1.3(a), the crosssection $V$ may be an arbitrary representation of the group $\Sigma_{2 m}$. In particular, the symbol $R$ is used to denote the abstract group element $R \in \Sigma_{2 m}$, the action of $R$ on the representation space $V$, and the induced action of $R$ on $\Gamma \times V \times S^{1}$.

(b) Although the index $m$ is well defined, the elements $\delta_{0} \in \Delta$ and $\zeta \in \mathrm{L} Z(\Sigma)$ are not in general well defined. It is natural to demand that $\delta_{0}$ has the least order possible. In many examples, this enables a well-defined choice of $\sigma$ and hence of $\delta_{0}, \exp \zeta$ and so on (but $\zeta$ itself is still not well defined).

(c) In general, we cannot hope to obtain $m$-periodicity in Theorem 4.12. However, let $\delta_{0}, Q$ and $Z$ be as in the proof of Theorem 4.12. If $\delta_{0} Q^{m} \in Z^{0}$, then we obtain an $m$-periodic bundle

$$
U \cong \frac{\left(\Gamma \times{ }_{\Delta} V\right) \times S^{1}}{\mathbb{Z}_{m}} \cong \frac{\Gamma \times V \times S^{1}}{\Sigma_{m}}
$$

where $S^{1}=\mathbb{R} / m \mathbb{Z}$.

(d) The derivation of the bundle in Theorem 4.12 involves passing to a comoving frame in the physical variables $(\gamma \mapsto \gamma \exp (-\theta \zeta))$ and simultaneously passing to a comoving frame in the phase space variables $(v \mapsto \exp (-\theta \eta) v)$.

If the relative periodic solution $\mathcal{P}$ is a discrete rotating wave then there is no need to go into a comoving frame, and Theorem 4.12 applies with $\zeta=0, \beta=\sigma$, and $\Sigma_{m}=\Sigma$. In particular, $\Sigma_{2 m}$ is precisely the group $\Sigma_{0}$ introduced in Vanderbauwhede $[\mathbf{3 3}, \mathbf{3 4}]$ for studying period doubling bifurcation (see also $[\mathbf{2 2}, \mathbf{2 6}]$ ). Hence Theorem 4.12 clarifies the role of $\Sigma$ and $\Sigma_{0}$ in the approach of $[\mathbf{2 6}, \mathbf{2 9}, \mathbf{3 3}, \mathbf{3 4}]$ to bifurcation from discrete rotating waves. A discussion of these approaches can be found in [22].

When $\mathcal{P}$ is a modulated traveling wave, it follows easily from Theorem 4.12 that the action of $\Delta$ on the cross-section $V$ extends to an (unfaithful) action of $\Sigma$ : let $\sigma$ act as $R^{-1}$.

When $\mathcal{P}$ is a modulated rotating wave, it is not necessarily the case that the action of $\Delta$ on $V$ extends to an action of $\Sigma$. For example, suppose that $\Sigma=\mathbf{S O}(2), \Delta=\mathbb{Z}_{2}$, and let $V$ be the one-dimensional non-trivial representation of $\Delta$.

In general, the $2 k$-periodicity of the equations on the slice, achieved in Theorem 1.3, is not preserved. This is due to the fact that $k$ and $m$ are unrelated in general [21].

Example 4.14. Consider a relative periodic solution $\mathcal{P}$ with $\Gamma=\mathbf{O}(2), \Sigma=\mathbb{D}_{\ell}$, and $\Delta=\mathbb{Z}_{\ell}, \ell \geq 3$. A concrete example is given by 'pulsating waves' in two-dimensional convection; see [29]. Since $\Sigma$ contains reflections, $\mathcal{P}$ is a discrete rotating wave (so $\zeta=0$, $\beta=\sigma$ in Theorem 4.12). Observe that $k=m=n=2$. In addition, $\sigma$ is necessarily of order two, so that $\delta_{0}=$ id.

Assume that the cross-section $V$ is two dimensional and that we have a faithful action of $\Delta=\mathbb{Z}_{\ell}$ on $V$. We show that the bundle in Theorem 4.12 can be chosen to be $m$-periodic and not only $2 m$-periodic.

Let $Q, G$, and $Z$ be as in the proof of Theorem 4.12. Since $Q$ is orthogonal, $Q$ is either a rotation or reflection on $V$. If $Q$ is a reflection, then $\delta_{0} Q^{m}=Q^{2}=I$. If $Q$ is a rotation, then $Z^{0}=Z=\mathbf{S O}(2)$, so that $\delta_{0} Q^{m}=Q^{2} \in Z^{0}$. Either way, it follows that 
Remark 4.13(c) applies, and the 4-periodic bundle in Theorem 4.12 reduces to a 2-periodic bundle.

\section{Proof of Proposition 1.2}

Throughout this section, $\Gamma$ is a finite-dimensional Lie group, $\Delta \subset \Gamma$ is a compact subgroup, and $\sigma$ lies in the normalizer $N(\Delta)$ of $\Delta$. The centralizer of $\Delta$ is denoted by $Z(\Delta)$. Each element $\sigma \in N(\Delta)$ defines an automorphism $\phi \in \operatorname{Aut}(\Delta)$ given by $\phi(\delta)=\sigma^{-1} \delta \sigma$. Recall that $\sigma$ is defined only up to the coset $\sigma \Delta$.

The main aim of this section is to prove Proposition 1.2. In addition, the following result is required in $\S 6$.

Proposition 5.1. Suppose that $\Gamma$ is a matrix group, $\Delta$ is a compact subgroup, and $\sigma_{0} \in N(\Delta)$. Then there is an element $\sigma \in \sigma_{0} \Delta$ such that $\sigma^{k} \in Z(\Delta)$ for some $k \geq 1$.

In $\S 5.1$, we prove a result about the relationship between $N(\Delta)$ and $Z(\Delta)$. In $\S \S 5.2$ and 5.3, we prove Propositions 5.1 and 1.2.

5.1. Normalizers and centralizers. Suppose that $\Gamma$ is a finite-dimensional Lie group with compact subgroup $\Delta$. Field [12, Proposition 3.2] showed that $N(\Delta)^{0}=\Delta^{0} Z(\Delta)^{0}$. We give a different proof of this result. (The ideas in this proof are required in Lemma 5.3 below.)

Lemma 5.2. Suppose that $\Gamma$ is a finite-dimensional Lie group and that $\Delta \subset \Gamma$ is a compact subgroup. Then:

(a) $\mathrm{L} N(\Delta)=\mathrm{L} \Delta+\mathrm{L} Z(\Delta)$;

(b) $\quad N(\Delta)^{0}=\Delta^{0} Z(\Delta)^{0}$.

Proof. The adjoint action of $\Delta$ on $\mathrm{L} \Gamma$ restricts to a representation of $\Delta$ on $\mathrm{L} N(\Delta)$. Moreover, the subalgebra $\mathrm{L} \Delta \subset \mathrm{L} N(\Delta)$ is a $\Delta$-invariant subspace. Since $\Delta$ is compact, we may choose a $\Delta$-invariant inner product on $\operatorname{LN}(\Delta)$. We then have the $\Delta$-invariant splitting

$$
\mathrm{L} N(\Delta)=\mathrm{L} \Delta \oplus(\mathrm{L} \Delta)^{\perp}
$$

Let $\eta \in(\mathrm{L} \Delta)^{\perp}$. To prove part (a), it is sufficient to prove that $\eta \in \mathrm{LZ}(\Delta)$. Let $\delta \in \Delta$. We show that $\operatorname{Ad}_{\delta} \eta=\eta$. By $\Delta$-invariance of $(\mathrm{L} \Delta)^{\perp}$, we have that $\operatorname{Ad}_{\delta} \eta-\eta \in(\mathrm{L} \Delta)^{\perp}$, so it remains to show that $\operatorname{Ad}_{\delta} \eta-\eta \in \mathrm{L} \Delta$. But $\eta \in \mathrm{L} N(\Delta)$ so that $\exp (t \eta) \delta^{-1} \exp (-t \eta) \in \Delta$. It follows that $\delta \exp (t \eta) \delta^{-1} \cdot \exp (-t \eta) \in \Delta$. Differentiating with respect to $t$ and setting $t=0$, we obtain $\operatorname{Ad}_{\delta} \eta-\eta \in \mathrm{L} \Delta$ as required.

Next, we prove part (b). Let $G$ consist of those elements $\gamma \in N(\Delta)^{0}$ such that $\gamma \in \Delta^{0} Z(\Delta)^{0}$. We show that $G$ is a non-empty open and closed subset of the connected component $N(\Delta)^{0}$ so that $G=N(\Delta)^{0}$.

Openness of $G$ is immediate from part (a), and it is an elementary argument to show that $G$ is non-empty and closed. Indeed, $G$ contains the identity and so is non-empty. To see that $G$ is closed, let $\left\{\gamma_{n}\right\}$ be a sequence in $G$ with $\gamma_{n} \rightarrow \gamma \in N(\Delta)^{0}$. Write $\gamma_{n}=\delta_{n} \tau_{n}$ where $\delta_{n} \in \Delta^{0}, \tau_{n} \in Z(\Delta)^{0}$. By compactness of $\Delta$, we may pass to a subsequence so that 
$\delta_{n} \rightarrow \delta \in \Delta^{0}$. Then $\tau_{n}=\delta_{n}^{-1} \gamma_{n} \rightarrow \delta^{-1} \gamma \in N(\Delta)^{0}$. Write $\tau=\lim _{n \rightarrow \infty} \tau_{n}=\delta^{-1} \gamma$. Since $Z(\Delta)^{0}$ is closed, $\tau \in Z(\Delta)^{0}$ so that $\gamma=\delta \tau \in \Delta^{0} Z(\Delta)^{0}$. Hence $\gamma \in G$ and $G$ is closed.

We require the following refinement of Lemma 5.2(a).

Lemma 5.3. Suppose that $\Gamma$ is a finite-dimensional Lie group and that $\Delta \subset \Gamma$ is a compact subgroup. Let $\sigma \in N(\Delta)$ and suppose that there is an integer $k \geq 1$ such that $\sigma^{k}=\exp \xi$, where $\xi \in \mathrm{L} N(\Delta)$ and $\operatorname{Ad}_{\sigma} \xi=\xi$. Then $\xi=\chi+\eta$ where $\chi \in \mathrm{L} \Delta$, $\eta \in \mathrm{LZ}(\Delta)$ and $\operatorname{Ad}_{\sigma} \chi=\chi, \operatorname{Ad}_{\sigma} \eta=\eta$.

Proof. As in the proof of Lemma 5.2, we have the unique decomposition $\xi=\chi+\eta$ where $\chi \in \mathrm{L} \Delta, \eta \in(\mathrm{L} \Delta)^{\perp} \subset \mathrm{L} Z(\Delta)$.

Let $\Sigma$ be the group generated by $\sigma$ and $\Delta$, and observe that $\mathrm{L} \Delta$ is $\Sigma$-invariant. If $\Sigma$ is compact, then the scalar product on $\mathrm{L} \Gamma$ can be chosen to be $\Sigma$-invariant. It then follows that $(\mathrm{L} \Delta)^{\perp}$ is $\Sigma$-invariant. In particular, we have the decomposition $\xi=\operatorname{Ad}_{\sigma} \xi=$ $\operatorname{Ad}_{\sigma} \chi+\operatorname{Ad}_{\sigma} \eta$ where $\operatorname{Ad}_{\sigma} \chi \in \mathrm{L} \Delta, \operatorname{Ad}_{\sigma} \eta \in(\mathrm{L} \Delta)^{\perp}$. By uniqueness of the decomposition, we have $\operatorname{Ad}_{\sigma} \chi=\chi, \operatorname{Ad}_{\sigma} \eta=\eta$ as required.

When $\Sigma$ is non-compact, the proof is more complicated. By Lemma 5.2 we have a preliminary decomposition $\xi=\tilde{\chi}+\tilde{\eta}$ where $\tilde{\chi} \in \mathrm{L} \Delta, \tilde{\eta} \in \mathrm{L} Z(\Delta)$. Consider the subspace $X=\mathrm{L} \Delta \oplus \mathbb{R}(\tilde{\eta})=\mathrm{L} \Delta+\mathbb{R}(\xi) \subset \mathrm{L} N(\Delta)$. Then $X$ is invariant under the adjoint actions of $\Delta$ and $\sigma$ and hence is a $\Sigma$-invariant subspace.

Let $(\cdot, \cdot)$ be any $\Delta$-invariant scalar product on $X$ and set

$$
\langle u, v\rangle=\sum_{j=0}^{k-1}\left(\operatorname{Ad}_{\sigma^{j}} u, \operatorname{Ad}_{\sigma^{j}} v\right) .
$$

We claim that $\langle u, v\rangle$ is $\Sigma$-invariant. We can then proceed as in the case when $\Sigma$ is compact to obtain a new decomposition $\xi=\chi+\eta$, this time with the required properties.

Since $\sigma \in N(\Delta)$, it follows that $\langle u, v\rangle$ is $\Delta$-invariant. It remains to show $\sigma$ invariance, specifically to show that $\left(\operatorname{Ad}_{\sigma^{k}} u, \operatorname{Ad}_{\sigma^{k}} v\right)=(u, v)$. Let $\delta=\exp \tilde{\chi} \in \Delta$, $\epsilon=\exp \tilde{\eta} \in Z(\Delta)$. Then it is sufficient, by $\Delta$-invariance of the inner product, to show that $\operatorname{Ad}_{\sigma^{k}}=\operatorname{Ad}_{\delta}$ on $X$.

Let $u \in \mathrm{L} \Delta$. Then $\operatorname{Ad}_{\sigma^{k}} u=\operatorname{Ad}_{\delta} \operatorname{Ad}_{\epsilon} u=\operatorname{Ad}_{\delta} u$ since $\epsilon \in Z(\Delta)$, and so $\operatorname{Ad}_{\sigma^{k}}=\operatorname{Ad}_{\delta}$ on $\mathrm{L} \Delta$. Since $\tilde{\eta} \in \mathrm{LZ}(\Delta)$ we have that $\operatorname{Ad}_{\delta} \tilde{\eta}=\tilde{\eta}$. At the same time, $[\xi, \tilde{\eta}]=[\tilde{\chi}, \tilde{\eta}]+[\tilde{\eta}, \tilde{\eta}]=0$ so that $\operatorname{Ad}_{\sigma^{k}} \tilde{\eta}=\operatorname{Ad}_{\exp \xi} \tilde{\eta}=\tilde{\eta}$. Therefore, $\operatorname{Ad}_{\sigma^{k}}$ and $\operatorname{Ad}_{\delta}$ are the identity on $\mathbb{R}(\tilde{\eta})$. This completes the proof that $\operatorname{Ad}_{\sigma^{k}}=\operatorname{Ad}_{\delta}$ on $X$.

5.2. Matrix groups and Proposition 5.1. We begin by proving Proposition 5.1 when $\Gamma$ is a compact Lie group. Then we obtain the result for matrix groups, and we give a counterexample for more general groups.

PROPOSITION 5.4. Suppose that $\Gamma$ is a compact Lie group, $\Delta$ is a compact subgroup, and $\sigma_{0} \in N(\Delta)$. Then there is an element $\sigma \in \sigma_{0} \Delta$ such that $\sigma^{k} \in Z(\Delta)$ for some $k \geq 1$. 
Proof. Compact Lie groups have finitely many connected components, so $\sigma_{0}^{k} \in(N(\Delta) \cap$ $\left.Z\left(\sigma_{0}\right)\right)^{0}$ for some $k \geq 1$. By surjectivity of the exponential map, $\sigma_{0}^{k}=\exp \xi$ where $\xi \in \mathrm{L} N(\Delta)$ and $\operatorname{Ad}_{\sigma_{0}} \xi=\xi$.

Let $\xi=\chi+\eta$ be the decomposition in Lemma 5.3 and define $\delta=\exp (\chi / k) \in \Delta$. Then $\sigma_{0}^{k}=\delta^{k} \exp \eta$, where $\exp \eta \in Z(\Delta)$. By Lemma 5.3, $\operatorname{Ad}_{\sigma_{0}} \chi=\chi$ and so $\sigma_{0}$ commutes with $\delta$. Hence $\sigma=\sigma_{0} \delta^{-1}$ satisfies $\sigma^{k}=\exp \eta \in Z(\Delta)$ as required.

Proof of Proposition 5.1. Since $\Gamma$ is a matrix group, $\Gamma$ acts faithfully on some finitedimensional vector space $V$. In particular, $\Delta$ acts faithfully on $V$. Let $\phi \in \operatorname{Aut}(\Delta)$ be the automorphism induced by $\sigma_{0}$, so that $\sigma_{0}^{-1} \delta \sigma_{0}=\phi(\delta)$. Equivalently, $\sigma_{0}^{-1}: V \rightarrow V$ is a twisted equivariant non-singular linear map $\left(\sigma_{0}^{-1} \delta=\phi(\delta) \sigma_{0}^{-1}\right)$. Choose a $\Delta$-invariant inner product on $V$. It follows from Proposition 2.1 that there is a twisted equivariant orthogonal map $Q: V \rightarrow V$. In particular,

$$
R^{-1} \delta R=\sigma_{0}^{-1} \delta \sigma_{0}=\phi(\delta),
$$

where $R=Q^{-1}$.

Let $G \subset \mathbf{O}(V)$ be the closed group generated by $\Delta$ and $R$. Then $G$ is a compact Lie group. It follows from Proposition 5.4 that there is an element $\delta_{0} \in \Delta$ such that $\widetilde{R}=R \delta_{0}$ satisfies $\widetilde{R}^{k} \in Z(\Delta)$ for some $k \geq 1$.

Now, define $\sigma=\sigma_{0} \delta_{0}$. Since $\sigma_{0}$ and $R$ induce the same automorphism $\phi \in \operatorname{Aut}(\Delta)$, it follows that $\sigma$ and $\widetilde{R}$ induce the same automorphism. Hence $\sigma^{k} \in Z(\Delta)$.

Remark 5.5. The hypothesis that $\Gamma$ is a matrix group can be weakened further to the assumption that $\Gamma$ has a finite-dimensional representation in which $\Delta$ acts faithfully.

Similarly, it is sufficient to assume that $\Delta$ has a finite-dimensional faithful representation $V$ for which there is a non-singular linear map $L: V \rightarrow V$ that is twisted equivariant with respect to the automorphism $\phi$.

Finally, we give a counterexample when $\Gamma$ is not a matrix group.

Example 5.6. Let $\Delta=T^{2}$. Then $\operatorname{Aut}(\Delta)$ is isomorphic to the group of $2 \times 2$ matrices with integer entries and determinant \pm 1 . In particular, $\mathbf{S L}(2, \mathbb{Z}) \subset \operatorname{Aut}(\Delta)$. Hence we can form the semidirect product $\Gamma=T^{2} \rtimes \mathbf{S L}(2, \mathbb{Z})$. Note that $\Gamma$ is a two-dimensional Lie group, $\Delta=T^{2}$ is a compact subgroup, and $N(\Delta)=\Gamma$. Moreover $Z(\Delta)=\Delta$. Now let $\sigma_{0}$ be any element of $\mathbf{S L}(2, \mathbb{Z})$ of infinite order. (For example, $\sigma_{0}=\left(\begin{array}{ll}2 & 1 \\ 1 & 1\end{array}\right)$.) Then $\sigma_{0}^{k} \notin Z(\Delta)$ for any $k \geq 1$. Since $\left(\sigma_{0} \Delta\right)^{k}=\sigma_{0}^{k} \Delta$, it follows that $\sigma^{k} \notin Z(\Delta)$ for any $\sigma \in \sigma_{0} \Delta$ and $k \geq 1$.

5.3. Algebraic groups and Proposition 1.2. As in the previous subsection, we prove Proposition 1.2 first for compact Lie groups, and then for algebraic groups.

PROPOSITION 5.7. Suppose that $\Gamma$ is a compact Lie group, $\Delta$ is a compact subgroup, and $\sigma_{0} \in N(\Delta)$. Let $\Sigma$ denote the closed subgroup of $\Gamma$ generated by $\Delta$ and $\sigma_{0}$. Then there is an element $\sigma \in \sigma_{0} \Delta$ such that $\sigma^{n} \in \exp \operatorname{L} Z(\Sigma)$ for some $n \geq 1$.

Proof. The proof is already implicit in the proof of Proposition 5.4 (with $n=k$ ). We showed there that $\sigma$ can be chosen so that $\sigma^{n}=\exp \eta \in \exp \operatorname{LZ}(\Delta)$. Furthermore, $\eta$ was constructed using Lemma 5.3, so that $\operatorname{Ad}_{\sigma_{0}} \eta=\eta$. Hence $\sigma^{n} \in \exp \operatorname{L}(\Sigma)$. 
Example 5.8. In contrast to the case for the integer $k$, Proposition 5.7 cannot be generalized to arbitrary matrix groups. For example, the group $\Gamma=\mathbb{Z}$ is a matrix group. Let $\Delta=\mathbf{1}$ and $\sigma_{0} \neq 0$. Then we are forced to take $\sigma=\sigma_{0}$, and no power lies in the connected component of the identity. In this example, $k=1$ but there is no finite integer $n$.

There are two ways in which compactness is utilized in the proof of Propositions 5.4 and 5.7. The first, which is highlighted in Examples 5.6 and 5.8, is that compact Lie groups have finitely many connected components. The second is that the exponential map $\exp : \mathrm{L} G \rightarrow G$ is surjective for connected compact Lie groups. This property may fail for connected but non-compact Lie groups, an example being $G=\mathbf{S L}(2, \mathbb{R})$; see Remark 4.8. The proof of Proposition 5.7 can be revised to take account of elements for which some sufficiently high power lies in the image of the exponential map. However, even this property fails in general.

Example 5.9. Let $G$ be the universal cover of $\mathbf{S L}(2, \mathbb{R})$. (Topologically, $G \cong \mathbb{R}^{3}$.) Again, $G$ is a connected semisimple Lie group. However, it can be shown that there exist elements $g \in G$ for which $g^{n}$ is not in the image of the exponential map for all $n \geq 1$; see [27, p. 164].

A similar, but more computable example, is the universal cover $G$ of $\mathbf{S E}(2)$. Again $G$ is homeomorphic to $\mathbb{R}^{3}$, but now $G$ is a connected solvable Lie group with a semidirect product structure $G=\mathbb{R} \ltimes \mathbb{R}^{2}$ where multiplication is defined by

$$
\left(t_{1}, v_{1}\right)\left(t_{2}, v_{2}\right)=\left(t_{1}+t_{2}, e^{2 \pi i t_{1}} v_{2}+v_{1}\right) \text {. }
$$

An elementary computation shows that $(t, v)$ lies in the image of the exponential map if and only if $v=0$ or $t \notin \mathbb{Z}$. Let $g=(1, v)$ where $v \neq 0$. Then $g^{m}=(m, m v)$, so $g^{m}$ is not in the image of the exponential map for all $m \geq 1$.

It turns out that Proposition 5.7 can be generalized to the class of algebraic groups. We require the following lemma of Goto [18].

LemmA 5.10. Suppose that $G$ is an algebraic group and that $g \in G$. Then there is an integer $n$ such that $g^{n} \in \exp \mathrm{L} G$.

Proof. The centralizer $Z(g)$ is a subgroup of $G$ defined by linear equalities and hence is algebraic. Similarly, the center $C$ of $Z(g)$ is algebraic and hence has finitely many connected components. Of course, $g$ lies in $C$, and so $g^{n} \in C^{0}$ for some $n \geq 1$. Moreover, $C^{0}$ is a finite-dimensional connected abelian Lie group and hence exp : $\mathrm{LC} \rightarrow C^{0}$ is surjective. It follows that $g^{n} \in \operatorname{expLC} \subset \exp \mathrm{L} G$.

In fact, Goto [18] proves that when $G$ is algebraic, there is an integer $n$ (depending only on $G$ ) such that $g^{n} \in \exp L G$ for all $g \in G$. We do not require this stronger result in this paper.

Proof of Proposition 1.2. Since $\Delta$ is compact, it follows from [37, p. 282] that $\Delta$ is algebraic and moreover that $\Delta$ and $\Gamma$ are realized simultaneously as algebraic groups (by inclusions $\Delta \subset \Gamma \subset \mathbf{G L}(n))$. It is immediate then that the normalizer of $\Delta$ inside $\Gamma$ is algebraic. Hence $N(\Delta) \cap Z\left(\sigma_{0}\right)$ is algebraic. By Lemma 5.10, there is an integer $n \geq 1$ 
such that $\sigma_{0}^{n}=\exp \xi$ where $\xi \in \mathrm{L}\left(N(\Delta) \cap Z\left(\sigma_{0}\right)\right)$. Now proceed as in the proof of Proposition 5.7.

Remark 5.11. In general, we define two integers $k$ and $n$, where $k$ divides $n$, associated with the element $\sigma_{0} \in N(\Delta)$. Namely, an integer $k$ such that $\sigma^{k} \in Z(\Delta)$ for some $\sigma \in \sigma_{0} \Delta$, and an integer $n$ such that $\sigma^{n} \in \exp \operatorname{LZ}(\Sigma)$ for some $\sigma \in \sigma_{0} \Delta$. When $\Gamma$ is algebraic, we have shown that $\sigma$ can be chosen so that $n$ (and hence $k$ ) is finite.

Calculations are simplest when $\sigma$ is chosen so that $k$ and $n$ are as small as possible. However, we note that in general it is not possible to choose a single representative $\sigma$ that simultaneously minimizes $k$ and $n$. For example, let $\Delta=\mathbf{S O}(2) \rtimes \mathbb{Z}_{4}$ where $\mathbb{Z}_{4}$ is generated by an element $\tau$ of order four that induces the automorphism $\theta \mapsto-\theta$ on elements $\theta \in \mathbf{S O}(2)$. Let $\Gamma=\Sigma=\Delta \rtimes \mathbb{Z}_{2}$ where $\mathbb{Z}_{2}$ is generated by an element $\sigma_{0}$ of order two such that $\sigma_{0} \tau$ lies in the center of $\Gamma$. (In other words, $\sigma_{0}$ commutes with $\tau$ and induces the same automorphism as $\tau$ on $\mathbf{S O}(2)$.) Then the minimum values of $k$ and $n$ are $k=1$ (achieved by $\sigma_{0} \tau$ ) and $n=2$ (achieved by $\sigma_{0}$ ), but there is no element of $\sigma_{0} \Delta$ that simultaneously achieves $k=1$ and $n=2$.

\section{A generalization of Theorem 1.3}

In this section, we show that many aspects of bifurcation from a relative periodic solution are captured by the methods in this paper, even when $\Gamma$ is not an algebraic group. For example, when a traveling wave is discretized in space, the underlying symmetry group is generally a matrix group (often $\Gamma=\mathbb{Z}^{d}$ ) but not algebraic. We note that 'discrete traveling waves' occur in discrete models of spatially periodic media and in numerical simulations.

We begin by supposing that $\Gamma$ is a matrix group, and then we consider the case when $\Gamma$ is an arbitrary finite-dimensional Lie group. Throughout, we assume that $\Gamma$ acts smoothly and properly on a finite-dimensional manifold $M$, and that $\mathcal{P}$ is a relative periodic solution with compact spatial symmetry $\Delta$. As before, the spatiotemporal symmetry $\Sigma$ is the closed subgroup of $\Gamma$ generated by the spatial symmetry $\Delta$ together with an element $\sigma$.

Provided $\Gamma$ is a matrix group, it follows from Proposition 5.1 that $\sigma$ can be shown to satisfy $\sigma^{k} \in Z(\Delta)$ for some $k=1$. We form the semidirect product $\Delta \rtimes \mathbb{Z}_{2 k}$ by adjoining to $\Delta$ an element $Q$ of order $2 k$ as described in the introduction. We also form a semidirect product $\Delta \rtimes \mathbb{Z}$ by adjoining an element of infinite order (with the same multiplication).

THEOREM 6.1. Suppose that $\Gamma$ is a matrix group, and define $\Delta \rtimes \mathbb{Z}_{2 k}$ and $\Delta \rtimes \mathbb{Z}$ as above.

(a) There is a neighborhood $U$ of the relative periodic solution $\mathcal{P}$ such that

$$
U \cong \frac{\left(\Gamma \times{ }_{\Delta} V\right) \times \mathbb{R}}{\mathbb{Z}} \cong \frac{\Gamma \times V \times \mathbb{R}}{\Delta \rtimes \mathbb{Z}}
$$

where $V$ is a representation of the group $\Delta \rtimes \mathbb{Z}_{2 k}$, and the action of $\Delta \rtimes \mathbb{Z}$ on $\Gamma \times V \times \mathbb{R}$ is given by

$$
(\gamma, v, \theta) \mapsto\left(\gamma \delta^{-1}, \delta v, \theta\right), \quad \delta \in \Delta, \quad(\gamma, v, \theta) \mapsto\left(\gamma \sigma^{-1}, Q v, \theta+1\right) .
$$

(b) The equations on $U$ lift to $(\Delta \times \mathbb{Z})$-equivariant skew product equations on $\Gamma \times V \times \mathbb{R}$ of the form (after reparameterizing time)

$$
\dot{\gamma}=\gamma f_{\Gamma}(v, \theta), \quad \dot{v}=f_{V}(v, \theta), \quad \dot{\theta}=1,
$$


where $f_{\Gamma}: V \times \mathbb{R} \rightarrow \mathrm{L} \Gamma$ and $f_{V}: V \times \mathbb{R} \rightarrow V$ are smooth vector fields satisfying $f_{\Gamma}(0, \theta)=0, f_{V}(0, \theta)=0$, for all $\theta \in \mathbb{R}$.

Proof. We proceed as in $\S \S 2.2$ and 2.3, except that we do not pass to a comoving frame (so $\xi=0$ ). In particular, the twisted equivariant linear map $Q: V \rightarrow V$ is chosen to have order $2 k$.

We note (as in Remark 1.4) that $f_{\Gamma}$ and $f_{V}$ are general vector fields satisfying the conditions stated in Theorem 6.1.

COROllary 6.2. Assume the set-up of Theorem 6.1, and let $S^{1}=\mathbb{R} / 2 k \mathbb{Z}$. Then the skew product equations restricted to $V \times \mathbb{R}$ define a general smooth $\Delta \rtimes \mathbb{Z}_{2 k}$-equivariant vector field on $V \times S^{1}$ of the form

$$
\dot{v}=f_{V}(v, \theta), \quad \dot{\theta}=1,
$$

where $f_{V}(0, \theta)=0$ for all $\theta \in S^{1}$. The action of $\Delta \rtimes \mathbb{Z}_{2 k}$ is given by

$$
(v, \theta) \mapsto(\delta v, \theta), \quad \delta \in \Delta, \quad(v, \theta) \mapsto(Q v, \theta+1) .
$$

It follows that provided $\Gamma$ is a matrix group, modulo drifts along group orbits, bifurcation from a relative periodic solution with compact spatial symmetry $\Delta$ reduces to bifurcation from an isolated periodic solution with spatial symmetry $\Delta$ and spatiotemporal symmetry group $\Delta \rtimes \mathbb{Z}_{2 k}$.

Finally, we show that a similar result is true even when $\Gamma$ is not a matrix group, except that now the integer $k$ depends on the specific representation of $\Delta$ on the cross-section $V$.

THEOREM 6.3. Let $V$ be a $\Delta$-invariant cross-section to the relative periodic solution at $u_{0}$. Let $L: V \rightarrow V$ be the linear map defined, as in $\$ 2.2$, by $L=P_{0} \sigma^{-1}\left(\mathrm{D} \Phi_{1}\right) u_{0}$.

Then the element $\sigma$ can be chosen (in $\sigma \Delta$ ) so that for some $k \geq 1$ :

(a) $\sigma^{k} \delta v=\delta \sigma^{k} v$ for all $\delta \in \Delta, v \in V$; and

(b) there is a linear map $A: V \rightarrow V$ satisfying $A^{2 k}=I$ such that $L^{-1} A$ is equivariantly isotopic to the identity.

With these choices of $\sigma, k$, and $Q=A^{-1}$, the conclusions of Theorem 6.1 hold.

Proof. The actions of $\Delta$ and $L$ on $V$ generate a group $G$ acting linearly on $V$. In general, the action of $G$ is not faithful. However, the quotient of $G$ by the kernel of the action is a matrix group and Proposition 5.1 applies to this quotient. Hence $\sigma$ can be chosen so that part (a) holds for some $k$. (In other words, $\sigma^{k}$ lies in the centralizer of $\Delta$ modulo the kernel of the action of $\Delta$ on $V$.) Part (b) follows from Lemma 2.2.

We stress that, in contrast to the case when $\Gamma$ is a matrix group, the choices of $\sigma, k$, and $Q$ in Theorem 6.3 depend on the specific representation of $\Delta$ on $V$.

Remark 6.4. (a) This theorem enables us to study bifurcation from a relative periodic solution for any finite-dimensional Lie group $\Gamma$. In particular, modulo drifts along continuous group orbits, the entire bifurcation is reduced to bifurcation from an isolated periodic solution for the group $\Delta \rtimes \mathbb{Z}_{2 k}$ generated by the actions of $\Delta$ and $Q$ on $V$.

(b) It is a consequence of Theorem 6.3 that situations such as that in Example 5.6 do not arise in the context of relative periodic solutions. That is, the existence of the 
twisted equivariant non-singular linear map $L$ arising from the flow precludes certain representations of $\Delta$. Let $\Gamma=T^{2} \rtimes \mathbf{S L}(2, \mathbb{Z})$ and $\Delta=T^{2}$. Suppose that $\sigma \in \mathbf{S L}(2, \mathbb{Z})$ is an element whose eigenvalues are not roots of unity. Then $\Delta$ is forced to act trivially on the subspace $V$.

Acknowledgements. Claudia Wulff was supported by the Deutsche Forschungsgemeinschaft, J. S. W. Lamb by a Talent Stipendium of the Netherlands Organization for Scientific Research (NWO) and I. Melbourne in part by NSF Grant DMS-9704980.

\section{Appendix. Equivariant linear maps}

In the proof of Theorem 4.12, we exploited a standard result about non-singular equivariant linear maps, namely that the square of a non-singular equivariant linear map is connected to the identity by a path of such maps. Presumably this result is well known, but we give a proof in this appendix since a complete proof is hard to find elsewhere.

Suppose that $G$ is a compact Lie group acting orthogonally on a real vector space $X$ and let $\operatorname{Hom}(X)$ denote the space of equivariant linear maps. Let $Z(X) \subset \operatorname{Hom}(X)$ denote the group of equivariant non-singular linear maps, and let $Z(X)^{0}$ denote the connected component of the identity in $Z(X)$. In this appendix, we prove that $B^{2} \in Z(X)^{0}$ for all $B \in Z(X)$.

Let $V$ be a $G$-irreducible subspace of $X$. Then $\operatorname{Hom}(V)$ is a real division ring $\mathcal{D}$ isomorphic to $\mathbb{R}, \mathbb{C}$, or $\mathbb{H}$. The irreducible subspace $V$ is said to be of real, complex, or quaternionic type.

The space $X$ can be decomposed (non-uniquely) as a direct sum of irreducible subspaces. There is also a unique isotypic decomposition $X=\oplus W_{j}$, where each isotypic component $W_{j}$ is a direct sum of isomorphic irreducible subspaces and distinct isotypic components consist of distinct irreducible subspaces. We say that an isotypic component $W$ is of real, complex, or quaternionic type if $W$ consists of irreducible subspaces of real, complex, or quaternionic type.

An important property of the isotypic decomposition is that the isotypic components are preserved by any equivariant linear map. In other words, $\operatorname{Hom}(X)=\oplus \operatorname{Hom}\left(W_{j}\right)$. Next, let $W$ be a fixed isotypic component. If we write $W=\oplus_{1}^{m} V$, then the isomorphism $\operatorname{Hom}(V) \cong \mathcal{D}$ induces an isomorphism $\operatorname{Hom}(W) \cong M_{m}(\mathcal{D})$ where $M_{m}(\mathcal{D})$ is the space of $m \times m$ matrices with entries in $\mathcal{D}$. In particular, the eigenvalues of $B \in \operatorname{Hom}(W)$ are given by the eigenvalues of the corresponding matrix $a \in M_{m}(\mathcal{D})$ but with multiplicity $\operatorname{dim}_{\mathcal{D}} V$. Altogether, we have a complete description of $\operatorname{Hom}(X)$.

Proposition A.1. Suppose that $W$ is an isotypic component.

(a) If $W$ is of complex or quaternionic type, then $Z(W)$ is connected.

(b) If $W$ is of real type, then $Z(W)$ has two connected components.

Proof. The isomorphism $\operatorname{Hom}(W) \cong M_{m}(\mathcal{D})$ induces an isomorphism $Z(W) \cong$ $\mathbf{G L}(m, \mathcal{D})$. Of course, $\mathbf{G L}(m, \mathbb{R})$ has two connected components and $\mathbf{G L}(m, \mathbb{C})$ has one connected component, as can be seen easily from the Jordan normal forms for real and complex matrices. The corresponding result in the quaternionic case follows similarly from the Jordan normal form for quaternionic matrices which can be found in [35]. 
Corollary A.2. Suppose that $G$ acts orthogonally on $X$ and that $B \in Z(X)$. Then $B^{2} \in Z(X)^{0}$.

Proof. Let $X=\oplus W_{j}$ be the isotypic decomposition of $X$, and write $B=\oplus B_{j}$ where $B_{j} \in Z\left(W_{j}\right)$. By Proposition A.1, $B_{j}^{2} \in Z\left(W_{j}\right)^{0}$ and hence $B^{2} \in Z(X)^{0}$.

Finally, we note that the results in this appendix go through without change if we restrict to spaces of orthogonal equivariant linear maps.

\section{REFERENCES}

[1] C. D. Andereck, S. S. Liu and H. L. Swinney. Flow regimes in a circular Couette system with independently rotating cylinders. J. Fluid Mech. 164 (1986), 155-183.

[2] P. Ashwin and I. Melbourne. Noncompact drift for relative equilibria and relative periodic orbits. Nonlinearity 10 (1997), 595-616.

[3] A. Bayliss, B. J. Matkowsky and H. Riecke. Symmetries in modulated traveling waves in combustion: jumping ponies on a merry-go-round. Pattern Formation: Symmetry Methods and Applications (Fields Institute Communications, 5). Eds. J. Chadam and W. Langford. American Mathematical Society, Providence, RI, 1995, pp. 19-43.

[4] T. Bröcker and T. tom Dieck. Representations of Compact Lie Groups (Graduate Texts in Mathematics, 98). Springer, New York, 1985.

[5] R. Carter, G. Segal and I. MacDonald. Lectures on Lie Groups and Lie Algebras (London Mathematical Society, Student Texts, 32). Cambridge University Press, 1995.

[6] P. Chossat and M. Golubitsky. Iterates of maps with symmetry. SIAM J. Math. Anal. 19 (1988), 12591270.

[7] T. tom Dieck. Topologie. Walter de Gruyter, Berlin, 1991.

[8] E. A. Ermakova, A. M. Pertsov and E. E. Shnol. On the interaction of vortices in two-dimensional active media. Physica D 40 (1989), 185-190.

[9] B. Fiedler, B. Sandstede, A. Scheel and C. Wulff. Bifurcation from relative equilibria to non-compact group actions: Skew products, meanders, and drifts. Doc. Math. J. DMV 1 (1996), 479-505.

[10] B. Fiedler and D. V. Turaev. Normal forms, resonances, and meandering tip motions near relative equilibria of Euclidean group actions. Arch. Rat. Mech. Anal. 145 (1998), 129-159.

[11] M. J. Field. Equivariant dynamical systems. Trans. Amer. Math. Soc. 259 (1980), 185-205.

[12] M. J. Field. Local structure for equivariant dynamics. Singularity Theory and its Applications, Part II (Lecture Notes in Mathematics, 1463). Eds. M. Roberts and I. Stewart. Springer, New York, 1991, pp. $142-166$.

[13] M. J. Field. Symmetry breaking for equivariant maps. Algebraic Groups and Related Subjects (Australian Mathematical Society Lecture Series). Eds. G. Lehrer et al. Cambridge University Press, 1997, pp. 219253.

[14] M. Golubitsky, V. G. LeBlanc and I. Melbourne. Hopf bifurcation from rotating waves and patterns in physical space. J. Nonl. Sci. (2000) 69-101.

[15] M. Golubitsky, I. Stewart and D. Schaeffer. Singularities and Groups in Bifurcation Theory, Vol. II (Applied Mathematical Sciences, 69). Springer, Berlin, 1988.

[16] M. Gorman, C. F. Hamill, M. el Hamdi and K.A. Robbins. Rotating and modulated rotating states of cellular flames. Combustion Sci. Technol. 98 (1994), 25-35.

[17] M. Gorman and H. L. Swinney. Spatial and temporal characteristics of modulated waves in the circular Couette system. J. Fluid Mech. 117 (1982), 123-142.

[18] M. Goto. Index of the exponential map of a semialgebraic group. Amer. J. Math. 100 (1978), 837-843.

[19] J. Guckenheimer and P. Holmes. Nonlinear Oscillations, Dynamical Systems, and Bifurcations of Vector Fields (Applied Mathematical Sciences, 42). Springer, New York, 1990.

[20] M. Krupa. Bifurcations of relative equilibria. SIAM J. Math. Anal. 21 (1990), 1453-1486.

[21] J. S. W. Lamb and I. Melbourne. Bifurcation from discrete rotating waves. Arch. Rat. Mech. Anal. (1999), 229-270. 
[22] J. S. W. Lamb and I. Melbourne. Bifurcation from periodic solutions with spatiotemporal symmetry. Pattern Formation in Continuous and Coupled Systems (IMA, 115). Eds. M. Golubitsky, D. Luss and S. Strogatz. Springer, New York, 1999, pp. 175-191.

[23] J. S. W. Lamb, I. Melbourne and C. Wulff. General bifurcations from periodic solutions with spatiotemporal symmetry, including mode interactions and resonances, in preparation.

[24] J. S. W. Lamb and G. R. W. Quispel. Reversing $k$-symmetries in dynamical systems. Physica D 73 (1994), 277-304.

[25] G. Li, Q. Ouyang, V. Petrov and H. L. Swinney. Transition from simple rotating chemical spirals to meandering and traveling spirals. Phys. Rev. Lett. 77 (1996), 2105-2108.

[26] N. Nicolaisen and B. Werner. Some remarks on period doubling in systems with symmetry. Z. Angew. Math. Phys. 46 (1995), 566-579.

[27] A. L. Onishchik and E. B. Vinberg. Lie Groups and Lie Algebras III (Encyclopaedia of Mathematical Sciences, 42). Springer, 1991.

[28] R. S. Palais. On the existence of slices for actions of non-compact Lie groups. Ann. Math. 73 (1961), 295-323.

[29] A. M. Rucklidge and M. Silber. Bifurcations of periodic orbits with spatio-temporal symmetries. Nonlinearity 11 (1998), 1435-1455.

[30] B. Sandstede, A. Scheel and C. Wulff. Dynamics of spiral waves on unbounded domains using centermanifold reductions. J. Diff. Eq. 141 (1997), 122-149.

[31] B. Sandstede, A. Scheel, and C. Wulff. Bifurcations and dynamics of spiral waves. J. Nonlin. Sci. 9 (1999), 439-478.

[32] G. S. Skinner and H. L. Swinney. Periodic to quasiperiodic transition of chemical spiral rotation. Physica D 48 (1991), 1-16.

[33] A. Vanderbauwhede. Equivariant period doubling. Advanced Topics in the Theory of Dynamical Systems (Notes Rep. Math. Sci. Engrg., 6). Eds. G. Fusco et al. Academic Press, Boston, 1989, pp. 235-246.

[34] A. Vanderbauwhede. Period-doublings and orbit-bifurcations in symmetric systems. Dynamical Systems and Ergodic Theory (Banach Center Publ., 23). Polish Scientific Publishers, Warsaw, 1989, pp. 197-208.

[35] N. A. Wiegmann. Some theorems on matrices with real quaternionic elements. Canad. J. Math. 7 (1955), 191-201.

[36] A. T. Winfree. The Geometry of Biological Time (Biomathematics, 8). Springer, New York, 1980.

[37] D. P. Želobenko. Compact Lie Groups and their Representations (Transl. of Math. Monographs, 40). American Mathematical Society, Providence, RI, 1973.

[38] V. S. Zykov. Cycloidal circulation of spiral waves in an excitable medium. Biofizika 31 (1986), 862-865. 